\title{
On an Intermediate Field Capturing Riemann solver based on a Parabolic viscosity matrix for the two-layer shallow water system
}

\author{
E. D. Fernández-Nieto ${ }^{*}$ M. J. Castro Díaz ${ }^{\dagger} \&$ C. Parés ${ }^{\ddagger}$
}

\begin{abstract}
The goal of this article is to design a new approximate Riemann solver for the two-layer shallow water system which is fast compared to Roe schemes and accurate compared to Lax-Friedrichs, FORCE, or GFORCE schemes (see [14]). This Riemann solver is based on a suitable decomposition of a Roe matrix (see [27]) by means of a parabolic viscosity matrix (see [16]) that captures some information concerning the intermediate characteristic fields. The corresponding first order numerical scheme, which is called IFCP (Intermediate Field Capturing Parabola) is linearly $L^{\infty}$-stable, well-balanced, and it doesn't require an entropy-fix technique. Some numerical experiments are presented to compare the behavior of this new scheme with Roe and GFORCE methods.
\end{abstract}

Short title : IFCP solver for the the two-layer SWS.

Keywords : Finite Volume Method, path-conservative, two-layer shallow water.

Subject Classifications : AMS (MOS) : 65N06, 76B15, 76M20, 76N99.

\section{Introduction}

We consider the system of partial differential equations governing the one-dimensional flow of two superposed immiscible layers of shallow water fluids studied in [12]:

\footnotetext{
*Departamento de Matemática Aplicada I, Universidad de Sevilla. E.T.S. Arquitectura. Avda, Reina Mercedes, s/n. 41012 Sevilla, Spain (edofer@us.es).

†Dpto. Análisis Matemático, Universidad de Málaga, Campus Teatinos s/n, 29071 Málaga, Spain (castro@anamat.cie.uma.es)

†Dpto. Análisis Matemático, Universidad de Málaga, Campus Teatinos s/n, 29071 Málaga, Spain (pares@anamat.cie.uma.es)
} 


$$
\left\{\begin{array}{l}
\frac{\partial h_{1}}{\partial t}+\frac{\partial q_{1}}{\partial x}=0 \\
\frac{\partial q_{1}}{\partial t}+\frac{\partial}{\partial x}\left(\frac{q_{1}^{2}}{h_{1}}+\frac{g}{2} h_{1}^{2}\right)=-g h_{1} \frac{\partial h_{2}}{\partial x}+g h_{1} \frac{d H}{d x} \\
\frac{\partial h_{2}}{\partial t}+\frac{\partial q_{2}}{\partial x}=0 \\
\frac{\partial q_{2}}{\partial t}+\frac{\partial}{\partial x}\left(\frac{q_{2}^{2}}{h_{2}}+\frac{g}{2} h_{2}^{2}\right)=-\frac{\rho_{1}}{\rho_{2}} g h_{2} \frac{\partial h_{1}}{\partial x}+g h_{2} \frac{d H}{d x}
\end{array}\right.
$$

In these equations, index 1 makes reference to the upper layer and index 2 to the lower one. The fluid is assumed to occupy a straight channel with constant rectangular cross-section and constant width. The coordinate $x$ refers to the axis of the channel, $t$ is time, and $g$ is the gravity acceleration. $H(x)$ represents the depth function measured from a fixed level of reference (see Figure 1). Each layer is assumed to have a constant density, $\rho_{i}, i=1,2\left(\rho_{1}<\rho_{2}\right)$. The unknowns $q_{i}(x, t)$ and $h_{i}(x, t)$ represent respectively the mass-flow and the thickness of the $i$ th layer at the section of coordinate $x$ at time $t$.



Figure 1: Two-layer sketch: unknowns $h_{1}, h_{2}$ and bathimetry function $H$.

The numerical resolution of two-layer or multilayer shallow water systems has been object of an intense research during the last years: see for instance [1], [3], [5], [6], [7], [8], [9], [10], [11], [12], [13], [14], [17], [22], [24] ... This interest is due, on the one hand, to the applicability of these models to the simulation of stratified geophysical flows. On the other hand, they can be considered as a prototype of P.D.E. systems involving flux terms, source terms, nonconservative products, wet/dry fronts, appearance of regions of hyperbolicity loss, etc. Therefore, any significant progress in the design of good numerical schemes for these models is potentially interesting for other models sharing some of these difficulties.

Before presenting the goal of this article, let us mention some mathematical aspects of system (1.1). Notice first that it can be written in the following form:

$$
w_{t}+F(w)_{x}+B(w) \cdot w_{x}=S(w) H_{x},
$$


where

$$
\begin{gathered}
w(x, t)=\left[\begin{array}{c}
h_{1}(x, t) \\
q_{1}(x, t) \\
h_{2}(x, t) \\
q_{2}(x, t)
\end{array}\right], \quad F(w)=\left[\begin{array}{c}
q_{1} \\
\frac{q_{1}^{2}}{h_{1}}+\frac{g}{2} h_{1}^{2} \\
q_{2} \\
\frac{q_{2}^{2}}{h_{2}}+\frac{g}{2} h_{2}^{2}
\end{array}\right], \quad S(w)=\left[\begin{array}{c}
0 \\
g h_{1} \\
0 \\
g h_{2}
\end{array}\right], \\
B(w)=\left[\begin{array}{cccc}
0 & 0 & 0 & 0 \\
0 & 0 & g h_{1} & 0 \\
0 & 0 & 0 & 0 \\
g r h_{2} & 0 & 0 & 0
\end{array}\right],
\end{gathered}
$$

and $r=\rho_{1} / \rho_{2}$. The vector $w$ takes values in the set:

$$
\mathcal{O}=\left\{\left[h_{1}, q_{1}, h_{2}, q_{2}\right]^{T} \in \mathbb{R}^{4}, \quad h_{1} \geq 0, h_{2} \geq 0\right\},
$$

as the thickness of the layers may vanish in practical applications when one or the two layers disappear in part of the domain.

By adding to (1.2) the equation

$$
H_{t}=0,
$$

the system can be rewritten in the form:

$$
W_{t}+\mathcal{A}(W) \cdot W_{x}=0
$$

where $W$ is the augmented vector

$$
W=\left[\begin{array}{l}
w \\
H
\end{array}\right] \in \Omega=\mathcal{O} \times \mathbb{R},
$$

and $\mathcal{A}(W)$ is the $5 \times 5$ matrix whose block structure is given by:

$$
\mathcal{A}(W)=\left[\begin{array}{c|c}
A(w) & -S(w) \\
\hline 0 & 0
\end{array}\right]
$$

where

$$
A(w)=J(w)+B(w), \quad \text { being } J(w)=\frac{\partial F}{\partial w}(w) .
$$

The stationary solutions of (1.1) can be obtained by parameterizing with $x$ the integral curves of the characteristic field of $\mathcal{A}(W)$ associated to the null eigenvalue, which are given by:

$$
q_{1}=\text { constant }, \quad \frac{u_{1}^{2}}{2}-\frac{u_{2}^{2}}{2}+g^{\prime} h_{1}=\text { constant },
$$




$$
q_{2}=\text { constant }, \quad \frac{u_{1}^{2}}{2}+g\left(h_{1}+h_{2}-H\right)=\text { constant },
$$

where $u_{i}=q_{i} / h_{i}$ represents the averaged velocity of the $i$ th layer, $i=1,2$ and $g^{\prime}=(1-r) g$ is the reduced gravity . In particular, if $q_{1}=0, q_{2}=0$, we obtain the solutions:

$$
q_{1}=0, h_{1}(x)=\text { constant }, q_{2}=0, h_{2}(x)-H(x)=\text { constant },
$$

corresponding to water at rest. Another steady state solution corresponding to vacuum is given by:

$$
h_{i}=0, \quad q_{i}=0, \quad i=1,2 .
$$

The characteristic equation of $A(w)$ is:

$$
\left(\lambda^{2}-2 u_{1} \lambda+u_{1}^{2}-g h_{1}\right)\left(\lambda^{2}-2 u_{2} \lambda+u_{2}^{2}-g h_{2}\right)=r g^{2} h_{1} h_{2} .
$$

It is easy to check that the condition under which one of the eigenvalues vanishes is:

$$
G^{2}=F_{1}^{2}+F_{2}^{2}-(1-r) F_{1}^{2} F_{2}^{2}=1,
$$

where $G$ is the so-called composite Froude number, and $F_{i}$ for $i=1,2$ are the internal Froude numbers $\left(F_{i}^{2}=\frac{u_{i}^{2}}{g^{\prime} h_{i}}\right)$. When this condition is achieved at a section of coordinate $x$, the flow is said to be critical at this point and the section $x$ is called a control. When $G^{2}<1$, the flow is subcritical. Finally, when $G^{2}>1$, the flow is supercritical.

Observe that, when $r=0$, the eigenvalues are those corresponding to each layer separately. Therefore, when $r \cong 0$, the coupling terms do not affect the nature of the system in an essential manner.

The eigenvalues of $A$ can be classified in two external and two internal ones. The external eigenvalues, $\lambda_{\text {ext }}^{ \pm}$, are related to the propagation speed of barotropic perturbations and the internal ones $\lambda_{\text {int }}^{ \pm}$, to the propagation of baroclinic perturbations. In the case $r \cong 1$ and $u_{1} \cong u_{2}$ (which is the situation arising in many geophysical flows), a first-order approximation of the eigenvalues was given in [26]:

$$
\begin{aligned}
& \lambda_{\text {ext }}^{ \pm} \cong \frac{u_{1} h_{1}+u_{2} h_{2}}{h_{1}+h_{2}} \pm\left(g\left(h_{1}+h_{2}\right)\right)^{\frac{1}{2}} \\
& \lambda_{\text {int }}^{ \pm} \cong \frac{u_{1} h_{2}+u_{2} h_{1}}{h_{1}+h_{2}} \pm\left(g^{\prime} \frac{h_{1} h_{2}}{\left(h_{1}+h_{2}\right)}\left[1-\frac{\left(u_{1}-u_{2}\right)^{2}}{g^{\prime}\left(h_{1}+h_{2}\right)}\right]\right)^{\frac{1}{2}} .
\end{aligned}
$$

Notice that the approximation of $\lambda_{\text {int }}^{ \pm}$become complex when

$$
\frac{\left(u_{1}-u_{2}\right)^{2}}{g^{\prime}\left(h_{1}+h_{2}\right)}>1
$$

Therefore, the hyperbolicity of the system is expected to be lost when (1.11) is satisfied, provided that $r \cong 1$ and $u_{1} \cong u_{2}$. This hyperbolicity loss is related to the 
appearance of shear instabilities that may lead, in real flows, to intense mixing of the two layers. In this work, the numerical treatment of this situation is not considered: the system will be assumed to be strictly hyperbolic.

In most applications to geophysical flows, one has $\lambda_{\text {ext }}^{-}<0$ and $\lambda_{\text {ext }}^{+}>0$. Moreover, the internal eigenvalues depend on the reduced gravity $g^{\prime}$ and $g^{\prime} \ll 1$. As a consequence the absolute value of internal eigenvalues are significantly smaller than those of external ones, that is

$$
\left|\lambda_{\text {int }}^{ \pm}\right|<\left|\lambda_{\text {ext }}^{-}\right|, \quad\left|\lambda_{\text {int }}^{ \pm}\right|<\left|\lambda_{\text {ext }}^{+}\right| .
$$

Accordingly, the following ordering of the eigenvalues will be considered on the sequel:

$$
\lambda_{1}=\lambda_{\text {ext }}^{-}, \quad \lambda_{2}=\lambda_{\text {int }}^{-}, \quad \lambda_{3}=\lambda_{\text {int }}^{+}, \lambda_{4}=\lambda_{\text {ext }}^{+} .
$$

The fact that the internal eigenvalues are usually much smaller than the external ones implies that first order numerical schemes that only use information concerning the 1st and 4th characteristic fields are in general too diffusive for the simulation of internal waves (see the numerical tests of Section 4). On the other hand, methods that use explicitly the eigenstructure of $A$, as it is the case for Roe methods, are computationally expensive, as there is not an easy explicit expression of the eigenvalues and eigenvectors of this system. In this work, we present an approximate Riemann solver that uses some information concerning the internal eigenvalues without requiring the computation of the complete eigenstructure. The definition of the method, which is based on a suitable decomposition of a Roe matrix (see [27]) by means of a parabolic viscosity matrix (see [16]) is presented in the next section. The well-balanced properties of the numerical scheme are studied in Section 3. Finally, a set of numerical tests are shown in Section 4. The CPU time and the accuracy of the numerical results for the proposed solver are tested. The numerical results are also compared with those given by the Roe (see [24]) and the GFORCE (see [14]) methods.

\section{Numerical schemes}

The solutions of (1.4) may develop discontinuities and, due to the non-divergence form of the equations, the notion of weak solution in the sense of distributions cannot be used. The theory introduced by Dal Maso, LeFloch, and Murat [15] is followed here to define weak solutions of (1.4). This theory allows one to define the nonconservative product $\mathcal{A}(W) \cdot W_{x}$ as a bounded measure provided a family of Lipschitz continuous paths $\Phi:[0,1] \times \Omega \times \Omega \rightarrow \Omega$ is prescribed, which must satisfy certain natural regularity conditions. Here, the family of straight segments is considered:

$$
\Phi\left(s ; W_{L}, W_{R}\right)=W_{L}+s\left(W_{R}-W_{L}\right) .
$$


We consider here path-conservative numerical schemes in the sense defined in [23], that is, numerical schemes of the general form:

$$
W_{i}^{n+1}=W_{i}^{n}-\frac{\Delta t}{\Delta x}\left(\mathcal{D}_{i-1 / 2}^{+}+\mathcal{D}_{i+1 / 2}^{-}\right),
$$

where $\Delta x$ and $\Delta t$ are, for simplicity, assumed to be constant; $W_{i}^{n}$ is the approximation provided by the numerical scheme of the cell average of the exact solution at the $i$ th cell, $I_{i}=\left[x_{i-1 / 2}, x_{i+1 / 2}\right]$ at the $n$-th time level $t^{n}=n \Delta t$, and

$$
\mathcal{D}_{i+1 / 2}^{ \pm}=\mathcal{D}^{ \pm}\left(W_{i}^{n}, W_{i+1}^{n}\right)
$$

where $\mathcal{D}^{-}$and $\mathcal{D}^{+}$are two Lipschitz continuous functions from $\Omega \times \Omega$ to $\Omega$ satisfying:

$$
\mathcal{D}^{ \pm}(W, W)=0, \quad \forall W \in \Omega
$$

and for every $W_{L}, W_{R} \in \Omega$,

$$
\mathcal{D}^{-}\left(W_{L}, W_{R}\right)+\mathcal{D}^{+}\left(W_{L}, W_{R}\right)=\int_{0}^{1} \mathcal{A}\left(\Phi\left(s ; W_{L}, W_{R}\right)\right) \frac{\partial \Phi}{\partial s}\left(s ; W_{L}, W_{R}\right) d s .
$$

These conditions provide a generalization of the concept of conservative scheme introduced by Lax for systems of conservation laws. In particular, in the case of the two-layer shallow water system, a path-conservative numerical scheme is conservative in the sense of Lax for the mass conservation equations.

An important difficulty related to the convergence of the numerical solutions appears: even if the correct family of paths is chosen, the limits of the numerical solutions may be weak solutions corresponding to a definition different from the one corresponding to the prescribed family. In fact, whenever a numerical scheme having some numerical viscosity is applied to a nonconservative system, the shocks appearing at the limits of the numerical solutions are limits of the viscous profiles of the modified equation whose viscous terms are different in general to the physical one and may lead thus to different jump conditions. This fact, which is strongly related to the difficulties of convergence appearing when a nonconservative method is applied to a system of conservation laws (see [21]) has been analyzed in [11] for nonconservative systems when a path-conservative mehtod is applied. Recently, a new example illustrating the difficulties discussed in [21] has been studied in [2]: the authors show that the difficulties of convergence are still present when a system of conservation laws is discretized by means of a nonconservative method which is path-conservative for a nonconservative reformulation of the system.

The numerical schemes considered in this work are based on a generalized Roe matrix for (1.4) as defined by Toumi in [27]: given a family of paths $\Phi$, a function $\mathcal{A}_{\Phi}: \Omega \times \Omega \mapsto \mathcal{M}_{5 \times 5}(\mathbb{R})$ is called a Roe linearization if it satisfies the following properties:

- for any $W_{L}, W_{R} \in \Omega, \mathcal{A}_{\Phi}\left(W_{L}, W_{R}\right)$ has 5 distinct real eigenvalues, 
- for every $W \in \Omega$,

$$
\mathcal{A}_{\Phi}(W, W)=\mathcal{A}(W)
$$

- for any $W_{L}, W_{R} \in \Omega$,

$$
\mathcal{A}_{\Phi}\left(W_{L}, W_{R}\right) \cdot\left(W_{R}-W_{L}\right)=\int_{0}^{1} \mathcal{A}\left(\Phi\left(s ; W_{L}, W_{R}\right)\right) \frac{\partial \Phi}{\partial s}\left(s ; W_{L}, W_{R}\right) d s
$$

Following [24], we consider Roe linearizations $\mathcal{A}_{\Phi}\left(W_{L}, W_{R}\right)$ of the form:

$$
\mathcal{A}_{\Phi}\left(W_{L}, W_{R}\right)=\left[\begin{array}{c|c}
A_{\Phi}\left(w_{L}, w_{R}\right) & -S_{\Phi}\left(w_{L}, w_{R}\right) \\
\hline 0 & 0
\end{array}\right]
$$

where

$$
A_{\Phi}\left(w_{L}, w_{R}\right)=J\left(w_{L}, w_{R}\right)+B_{\Phi}\left(w_{L}, w_{R}\right) .
$$

Here, $J\left(w_{L}, w_{R}\right)$ is a Roe linearization of the Jacobian of the flux $F$ in the usual sense:

$$
J\left(w_{L}, w_{R}\right) \cdot\left(w_{R}-w_{L}\right)=F\left(w_{R}\right)-F\left(w_{L}\right) ;
$$

$B_{\Phi}\left(w_{L}, w_{R}\right)$ is a matrix satisfying:

$$
B_{\Phi}\left(w_{L}, w_{R}\right) \cdot\left(w_{R}-w_{L}\right)=\int_{0}^{1} B\left(\Phi\left(s ; W_{L}, W_{R}\right)\right) \frac{\partial \Phi_{[1, \cdots, 4]}}{\partial s}\left(s ; W_{L}, W_{R}\right) d s ;
$$

and $S_{\Phi}\left(w_{L}, w_{R}\right)$ is a vector satisfying:

$$
S_{\Phi}\left(w_{L}, w_{R}\right)\left(H_{R}-H_{L}\right)=\int_{0}^{1} S\left(\Phi\left(s ; W_{L}, W_{R}\right)\right) \frac{\partial \Phi_{5}}{\partial s}\left(s ; W_{L}, W_{R}\right) d s .
$$

It can be easily shown that, if (2.7)-(2.9) are satisfied, then the matrix defined by (2.5)-(2.6) is a Roe linearization provided it has 5 different real eigenvalues.

The Roe linearization considered here is the following: given two states

$$
W_{L}=\left[\begin{array}{c}
h_{1}^{L} \\
q_{1}^{L} \\
h_{2}^{L} \\
q_{2}^{L} \\
H_{L}
\end{array}\right], \quad W_{R}=\left[\begin{array}{c}
h_{1}^{R} \\
q_{1}^{R} \\
h_{2}^{R} \\
q_{2}^{R} \\
H_{R}
\end{array}\right]
$$

the matrices $J\left(w_{L}, w_{R}\right), B_{\Phi}\left(w_{L}, w_{R}\right)$, and the vector $S_{\Phi}\left(w_{L}, w_{R}\right)$ are defined by:

$$
J\left(w_{L}, w_{R}\right)=\left[\begin{array}{cccc}
0 & 1 & 0 & 0 \\
-\left(\hat{u}_{1}\right)^{2}+\left(\hat{c}_{1}\right)^{2} & 2 \hat{u}_{1} & 0 & 0 \\
0 & 0 & 0 & 1 \\
0 & 0 & -\left(\hat{u}_{2}\right)^{2}+\left(\hat{c}_{2}\right)^{2} & 2 \hat{u}_{2}
\end{array}\right],
$$




$$
\begin{gathered}
B_{\Phi}\left(w_{L}, w_{R}\right)=\left[\begin{array}{cccc}
0 & 0 & 0 & 0 \\
0 & 0 & \left(\hat{c}_{1}\right)^{2} & 0 \\
0 & 0 & 0 & 0 \\
r\left(\hat{c}_{2}\right)^{2} & 0 & 0 & 0
\end{array}\right] \\
S_{\Phi}\left(w_{L}, w_{R}\right)=\left(\begin{array}{c}
0 \\
\left(\hat{c}_{1}\right)^{2} \\
0 \\
\left(\hat{c}_{2}\right)^{2}
\end{array}\right)
\end{gathered}
$$

where

$$
\hat{u}_{k}=\frac{\sqrt{h_{k}^{L}} u_{k}^{L}+\sqrt{h_{k}^{R}} u_{k}^{R}}{\sqrt{h_{k}^{L}}+\sqrt{h_{k}^{R}}}, \quad \hat{c}_{k}=\sqrt{g \frac{h_{k}^{L}+h_{k}^{R}}{2}}, \quad k=1,2
$$

with

$$
u_{k}^{L}=\frac{q_{k}^{L}}{h_{k}^{L}}, \quad u_{k}^{R}=\frac{q_{k}^{R}}{h_{k}^{R}}, \quad k=1,2 .
$$

Once the linearization has been chosen, the Roe scheme is given by (2.1) with:

$$
\mathcal{D}_{i+1 / 2}^{ \pm}=\mathcal{A}_{\Phi}^{ \pm}\left(W_{i}^{n}, W_{i+1}^{n}\right) \cdot\left(W_{i+1}^{n}-W_{i}^{n}\right),
$$

where

$$
\mathcal{A}_{\Phi}^{ \pm}\left(W_{L}, W_{R}\right)=\mathcal{K}_{\Phi}\left(W_{L}, W_{R}\right) \mathcal{L}_{\Phi}^{ \pm}\left(W_{L}, W_{R}\right) \mathcal{K}_{\Phi}^{-1}\left(W_{L}, W_{R}\right) .
$$

Here, $\mathcal{L}_{\Phi}^{ \pm}\left(W_{L}, W_{R}\right)$ represents the diagonal matrix whose coefficients are the positive/negative part of the eigenvalues of $\mathcal{A}_{\Phi}\left(W_{L}, W_{R}\right)$, and $\mathcal{K}_{\Phi}\left(W_{L}, W_{R}\right)$ is the matrix whose $i$ th column is an eigenvector associated to the $i$ th eigenvalue.

It can be shown (see [24]) that the numerical scheme can be written in the variables $w$ as follows:

$$
w_{i}^{n+1}=w_{i}^{n}-\frac{\Delta t}{\Delta x}\left(D_{i-1 / 2}^{+}+D_{i+1 / 2}^{-}\right)
$$

being

$$
\begin{aligned}
D_{i+1 / 2}^{ \pm}= & \frac{1}{2}\left(F\left(w_{i+1}^{n}\right)-F\left(w_{i}^{n}\right)+B_{i+1 / 2}\left(w_{i+1}^{n}-w_{i}^{n}\right)\right. \\
& -S_{i+1 / 2}\left(H_{i+1}-H_{i}\right) \\
& \left. \pm\left|A_{i+1 / 2}\right|\left(w_{i+1}^{n}-w_{i}^{n}-A_{i+1 / 2}^{-1} S_{i+1 / 2}\left(H_{i+1}-H_{i}\right)\right)\right),
\end{aligned}
$$

and $B_{i+1 / 2}=B_{\Phi}\left(w_{i}^{n}, w_{i+1}^{n}\right), S_{i+1 / 2}=S_{\Phi}\left(w_{i}^{n}, w_{i+1}^{n}\right), A_{i+1 / 2}=A_{\Phi}\left(w_{i}^{n}, w_{i+1}^{n}\right)$. Here $\left|A_{\Phi}\left(w_{L}, w_{R}\right)\right|=K_{\Phi}\left(w_{L}, w_{R}\right)\left|L_{\Phi}\left(w_{L}, w_{R}\right)\right| K_{\Phi}^{-1}\left(w_{L}, w_{R}\right)$, where $\left|L_{\Phi}\left(w_{L}, w_{R}\right)\right|$ represents the diagonal matrix whose coefficients are the absolute values of the eigenvalues of $A_{\Phi}\left(w_{L}, w_{R}\right)$, and $K_{\Phi}\left(w_{L}, w_{R}\right)$ is the matrix whose $i$ th column is an eigenvector associated to the $i$ th eigenvalue. 
This expression of the Roe scheme suggests the following generalization

$$
\begin{aligned}
D_{i+1 / 2}^{ \pm}= & \frac{1}{2}\left(F\left(w_{i+1}^{n}\right)-F\left(w_{i}^{n}\right)+B_{i+1 / 2}\left(w_{i+1}^{n}-w_{i}^{n}\right)\right. \\
& -S_{i+1 / 2}\left(H_{i+1}-H_{i}\right) \\
& \left. \pm Q_{i+1 / 2}\left(w_{i+1}^{n}-w_{i}^{n}-A_{i+1 / 2}^{-1} S_{i+1 / 2}\left(H_{i+1}-H_{i}\right)\right)\right)
\end{aligned}
$$

where $Q_{i+1 / 2}=Q_{\Phi}\left(w_{i}^{n}, w_{i+1}^{n}\right)$ is any numerical viscosity matrix. For instance, the choice:

$$
Q_{\Phi}\left(w_{L}, w_{R}\right)=(1-\omega) \frac{\Delta x}{\Delta t} I d+\omega \frac{\Delta t}{\Delta x} A_{\Phi}^{2}\left(w_{L}, w_{R}\right),
$$

corresponds to the family of numerical schemes introduced in [14]. This family contains, as particular cases, a well-balanced extension of the Lax-Friedrichs $(\omega=0)$, Lax-Wendroff $(\omega=1)$, FORCE $\left(\omega=\frac{1}{2}\right)$, and $\operatorname{GFORCE}\left(\omega=\frac{1}{1+C F L}\right)$ methods, where:

$$
C F L=\left(\max \left\{\left|\lambda_{l, i+1 / 2}\right|, 1 \leq l \leq 4, i \in \mathbb{Z}\right\} \frac{\Delta t}{\Delta x}\right) \in(0,1],
$$

being $\lambda_{l, i+1 / 2}$ for $l=1, \ldots, 4$ the eigenvalues of Roe matrix $A_{i+1 / 2}$.

The goal of this work is to introduce a new viscosity matrix leading to a numerical scheme whose accuracy is close to that of the Roe scheme and whose computational cost is similar to the FORCE o GFORCE methods. In order to introduce such as viscosity matrix, let us first observe that the matrix $\left|A_{\Phi}\left(w_{L}, w_{R}\right)\right|$ can be rewritten as follows (see [16]):

$$
\left|A_{\Phi}\left(w_{L}, w_{R}\right)\right|=\sum_{j=0}^{3} \alpha_{j} A_{\Phi}^{j}\left(w_{L}, w_{R}\right),
$$

where $\alpha_{j}, j=0, \cdots, 3$ are defined in terms of the eigenvalues $\lambda_{j}, j=1, \cdots, 4$ of $A_{\Phi}\left(w_{L}, w_{R}\right)$, by solving the linear system:

$$
\left(\begin{array}{cccc}
1 & \lambda_{1} & \lambda_{1}^{2} & \lambda_{1}^{3} \\
1 & \lambda_{2} & \lambda_{2}^{2} & \lambda_{2}^{3} \\
1 & \lambda_{3} & \lambda_{3}^{2} & \lambda_{3}^{3} \\
1 & \lambda_{4} & \lambda_{4}^{2} & \lambda_{4}^{3}
\end{array}\right)\left(\begin{array}{l}
\alpha_{0} \\
\alpha_{1} \\
\alpha_{2} \\
\alpha_{3}
\end{array}\right)=\left(\begin{array}{c}
\left|\lambda_{1}\right| \\
\left|\lambda_{2}\right| \\
\left|\lambda_{3}\right| \\
\left|\lambda_{4}\right|
\end{array}\right)
$$

Note that (2.20) has an unique solution provided that $\lambda_{i} \neq \lambda_{j}, i \neq j, i, j=1, \cdots, 4$. Observe that the explicit knowledge of the eigenvectors of $A_{\Phi}\left(w_{L}, w_{R}\right)$ is not needed to compute $\left|A_{\Phi}\left(w_{L}, w_{R}\right)\right|$ by using (2.19). Nevertheless, the CPU time required to compute it in this way is similar to the one needed to compute the complete eigenstructure of $A_{\Phi}\left(w_{L}, w_{R}\right)$.

We can extend this expression and consider the family of numerical schemes given by (2.16) with a parabolic viscosity matrix (see [16]) of the form:

$$
Q_{\Phi}\left(w_{L}, w_{R}\right)=\alpha_{0} I d+\alpha_{1} A_{\Phi}\left(w_{L}, w_{R}\right)+\alpha_{2} A_{\Phi}^{2}\left(w_{L}, w_{R}\right)
$$


where $\alpha_{j}, j=0,1,2$ are defined in terms of the eigenvalues of $A_{\Phi}\left(w_{L}, w_{R}\right)$. In fact, the numerical scheme defined by (2.16)-(2.17) is the particular case corresponding to $\alpha_{0}=(1-\omega) \frac{\Delta x}{\Delta t}, \alpha_{1}=0$, and $\alpha_{2}=\omega \frac{\Delta t}{\Delta x}$

Let us consider the viscosity matrix (2.21), where $\alpha_{j}, j=0,1,2$ are defined by:

$$
\left(\begin{array}{ccc}
1 & \lambda_{1} & \lambda_{1}^{2} \\
1 & \lambda_{4} & \lambda_{4}^{2} \\
1 & \chi_{\text {int }} & \chi_{\text {int }}^{2}
\end{array}\right)\left(\begin{array}{c}
\alpha_{0} \\
\alpha_{1} \\
\alpha_{2}
\end{array}\right)=\left(\begin{array}{c}
\left|\lambda_{1}\right| \\
\left|\lambda_{4}\right| \\
\left|\chi_{\text {int }}\right|
\end{array}\right)
$$

where

$$
\chi_{\text {int }}=\mathcal{S}_{\text {ext }} \max \left(\left|\lambda_{2}\right|,\left|\lambda_{3}\right|\right)
$$

with

$$
\mathcal{S}_{\text {ext }}= \begin{cases}\operatorname{sgn}\left(\lambda_{2}+\lambda_{3}\right), & \text { if }\left(\lambda_{2}+\lambda_{3}\right) \neq 0 \\ 1, & \text { otherwise }\end{cases}
$$

The expression of the coefficients $\alpha_{j}, j=0,1,2$ can be explicitly computed by solving (2.22):

$$
\begin{aligned}
& \alpha_{0}=\delta_{1} \lambda_{4} \chi_{i n t}+\delta_{4} \lambda_{1} \chi_{i n t}+\delta_{i n t} \lambda_{1} \lambda_{4}, \\
& \alpha_{1}=-\lambda_{1}\left(\delta_{4}+\delta_{i n t}\right)-\lambda_{4}\left(\delta_{1}+\delta_{i n t}\right)-\chi_{i n t}\left(\delta_{1}+\delta_{4}\right), \\
& \alpha_{2}=\delta_{1}+\delta_{4}+\delta_{i n t},
\end{aligned}
$$

where

$$
\delta_{1}=\frac{\left|\lambda_{1}\right|}{\left(\lambda_{1}-\lambda_{4}\right)\left(\lambda_{1}-\chi_{i n t}\right)}, \delta_{4}=\frac{\left|\lambda_{4}\right|}{\left(\lambda_{4}-\lambda_{1}\right)\left(\lambda_{4}-\chi_{i n t}\right)}, \quad \delta_{i n t}=\frac{\left|\chi_{i n t}\right|}{\left(\chi_{i n t}-\lambda_{1}\right)\left(\chi_{i n t}-\lambda_{4}\right)} .
$$

It can be proved that the numerical scheme defined by $(2.14)-(2.16)$, where $Q_{\Phi}\left(w_{L}, w_{R}\right)$ is given by (2.21)-(2.25) is linearly $L^{\infty}$-stable under the usual CFL condition (2.18) (see Appendix).

Remark 1. 1. Although it has been assumed that Roe matrices have 5 distinct real eigenvalues, notice that in practice the numerical scheme can be applied if the two following requirements are satisfied: (a) $\lambda_{1}, \lambda_{4}, \chi_{\text {int }}$ have to be different so that $\alpha_{i}, i=0,1,2$ are well defined, and (b) $\lambda_{i} \neq 0, i=1, \cdots, 4$ so that $A_{i+1+2}^{-1}$ is also well defined. Concerning (a), this property is always satisfied by the geophysical flows to be simulated and concerning (b), some techniques will be introduced in the next section to avoid this requirement. On the other hand, when the system loses its hyperbolic character, complex eigenvalues can appear in the Roe matrices. Although the numerical scheme can be easily adapted to be applied even in these situations, it is no more linearly $L^{\infty}$-stable: an additional strategy must be considered to stabilize the computations, as for example introducing some interface friction term or an intermediate layer. Some works in these directions are being carried out. 
2. Even if the $L^{\infty}$-stability of the previous numerical scheme is only theoretically ensured if the exact eigenvalues of $A_{\Phi}\left(w_{L}, w_{R}\right)$ are used, no instabilities are observed in practice when their first order approximations (1.9)-(1.10) are used instead. This is what makes this numerical scheme computationally fast when compared to the Roe method, while it is more accurate compared to the schemes defined by (2.17) as it uses some information concerning the internal eigenvalues.

3. Observe that, if one of internal eigenvalues vanishes, the viscosity matrix (2.21) does not have a null eigenvalue, as it happens with the viscosity matrix of the Roe scheme. Due to this, this new scheme does not require an entropy-fix technique to avoid the appearance of 'dog-leg' effects in transonic rarefactions, as it happens with the Roe method.

4. Although the numerical scheme (2.14)-(2.16), where $Q_{\Phi}\left(w_{L}, w_{R}\right)$ is given by (2.21)-(2.25) has been derived here for the two-layer shallow water system, its extension to some other nonconservative hyperbolic systems is straightforward. This is the case for instance of some two-phase fluid systems like the Pitman and Le model [25], or the submarine avalanches model proposed in [19].

\section{Well-balancing}

All the numerical schemes given by (2.14)-(2.16) are well-balanced for water at rest solutions. To see this notice that (2.16) can be written as follows:

$$
\begin{aligned}
D_{i+1 / 2}^{ \pm} & =\frac{1}{2}\left(A_{i+1 / 2}\left(w_{i+1}^{n}-w_{i}^{n}\right)-S_{i+1 / 2}\left(H_{i+1}-H_{i}\right)\right. \\
& \left. \pm Q_{i+1 / 2} A_{i+1 / 2}^{-1}\left(A_{i+1 / 2}\left(w_{i+1}^{n}-w_{i}^{n}\right)-S_{i+1 / 2}\left(H_{i+1}-H_{i}\right)\right)\right)
\end{aligned}
$$

and the chosen Roe matrix satisfies the equality

$$
A_{i+1 / 2}\left(w_{i+1}^{n}-w_{i}^{n}\right)=S_{i+1 / 2}\left(H_{i+1}-H_{i}\right),
$$

whenever $\left[w_{i}^{n}, H_{i}\right]^{T},\left[w_{i+1}^{n}, H_{i+1}\right]^{T}$ are two water at rest states with the same free surface and interface locations (see [24]).

Nevertheless, note that in the definition of (2.16) the term

$$
C=Q_{i+1 / 2} A_{i+1 / 2}^{-1} S_{i+1 / 2}\left(H_{i+1}-H_{i}\right),
$$

that can be interpreted as the upwinding part of the source term discretization, makes no sense if one of the eigenvalues of $A_{i+1 / 2}$ vanishes. In this case two eigenvalues of $\mathcal{A}_{\Phi}\left(W_{i}^{n}, W_{i+1}^{n}\right)$ vanish and the problem is said to be resonant. Resonant problems exhibit an additional difficulty, as weak solutions may not be uniquely determined by their initial data, and the limits of the numerical solutions may depend both on the family of paths and the numerical scheme itself. The analysis of this 
difficulty is beyond the scope of this article. According to our general purpose here, we would like to introduce some easy modifications of the schemes which avoid the difficulty related to the presence of $A_{i+1 / 2}^{-1}$.

In the case of Roe scheme, the following definition of $C$ may be used

$$
C=\operatorname{sgn}\left(A_{i+1 / 2}\right) S_{i+1 / 2}\left(H_{i+1}-H_{i}\right),
$$

where $\operatorname{sgn}\left(A_{i+1 / 2}\right)=K_{i+1 / 2} \operatorname{sgn}\left(L_{i+1 / 2}\right) K_{i+1 / 2}^{-1}$, being, $\operatorname{sgn}\left(L_{i+1 / 2}\right)$ the diagonal matrix whose coefficients are the signs of the eigenvalues of $A_{i+1 / 2}$, and $K_{i+1 / 2}=$ $K_{\Phi}\left(w_{i}^{n}, w_{i+1}^{n}\right)$.

If $Q_{\Phi}\left(w_{L}, w_{R}\right)$ is given by $(2.21)-(2.25), C$ reduces to

$C=\underbrace{\alpha_{0} A_{i+1 / 2}^{-1} S_{i+1 / 2}\left(H_{i+1}-H_{i}\right)}_{C_{0}}+\alpha_{1} S_{i+1 / 2}\left(H_{i+1}-H_{i}\right)+\alpha_{2} A_{i+1 / 2} S_{i+1 / 2}\left(H_{i+1}-H_{i}\right)$.

Observe that $C_{0}$ is the only term which is meaningless when $A_{i+1 / 2}$ is singular. We propose here two different techniques to get rid of this difficulty. The first one, following [14], consists in replacing $A_{i+1 / 2}^{-1}=A_{\Phi}^{-1}\left(w_{i+1}^{n}, w_{1}^{n}\right)$ by $A_{\Phi}^{-1}\left(w_{i}^{*, n}, w_{i+1}^{*, n}\right)$, where $w_{i}^{*}=\left[h_{1, i}, 0, h_{2, i}, 0\right]^{T}$. The resulting numerical scheme is exactly well-balanced for the water at rest solutions (1.5) and it is always well-defined. Numerical difficulties also appear when wet/dry fronts develop. Nevertheless, the techniques introduced for the Roe scheme in [10] can be easily extended to this new scheme. The resulting numerical scheme will be named IFCP hereafter.

The second strategy to deal with the difficulty related to resonant problems consists in using the generalized hydrostatic reconstruction introduced in [13] which is an extension of the technique introduced in [4] for the numerical treatment of the source term in the shallow water system. In the case of the two-layer shallow water system, the resulting numerical scheme reads as follows (see [13] for details):

$$
w_{i}^{n+1}=w_{i}-\frac{\Delta t}{\Delta x}\left(E_{i-1 / 2}^{+}+E_{i+1 / 2}^{-}\right),
$$

where

$$
\begin{aligned}
E_{i+1 / 2}^{-}= & F\left(w_{i+1 / 2}^{n,-}\right)-F\left(w_{i}^{n}\right)+D_{i+1 / 2}^{-} \\
& +\left(\begin{array}{c}
g h_{i, 1}^{n}\left(h_{i+1 / 2,2}^{n,-}-h_{i, 2}^{n}-\left(\bar{H}_{i+1 / 2}^{n,-}-H_{i}\right)\right) \\
0 \\
0
\end{array}\right)-\left(\begin{array}{c}
0 \\
\frac{g}{2}\left(h_{i+1 / 2,1}^{n,-}\right)^{2}-\frac{g}{2}\left(h_{i, 1}^{n}\right)^{2} \\
0 \\
\frac{g}{2}\left(h_{i+1 / 2,2}^{n,-}\right)^{2}-\frac{g}{2}\left(h_{i, 2}^{n}\right)^{2}
\end{array}\right)
\end{aligned}
$$


and

$$
\begin{aligned}
& E_{i+1 / 2}^{+}=F\left(w_{i+1}^{n}\right)-F\left(w_{i+1 / 2}^{n,+}\right)+D_{i+1 / 2}^{+}
\end{aligned}
$$

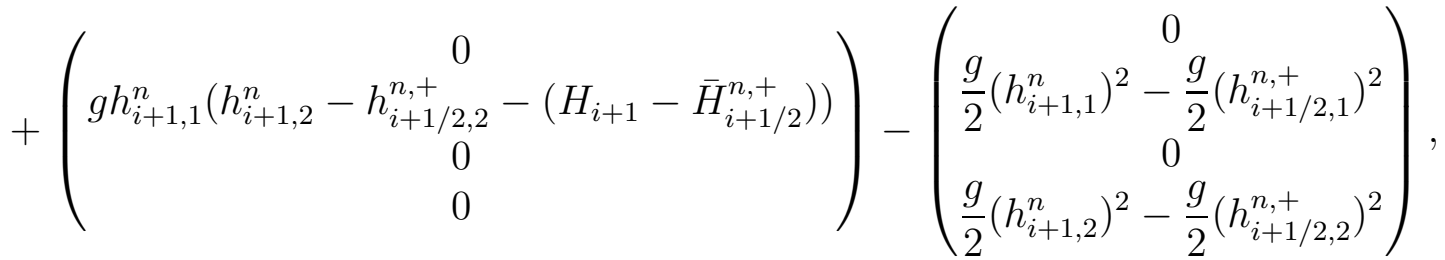

where $H_{i+1 / 2}=\min \left(H_{i}, H_{i+1}\right), \bar{H}_{i+1 / 2}^{n,+}=\max \left(H_{i+1}-h_{i+1,2}^{n}, H_{i+1 / 2}\right), \bar{H}_{i+1 / 2}^{n,-}=$ $\max \left(H_{i}-h_{i, 2}^{n}, H_{i+1 / 2}\right)$, and

$$
\begin{gathered}
w_{i+1 / 2}^{n,+}=\left[\begin{array}{c}
h_{i+1 / 2,1}^{n,+} \\
q_{i+1 / 2,1}^{n,+} \\
h_{i+1 / 2,2}^{n,+} \\
q_{i+1 / 2,2}^{n,+}
\end{array}\right]=\left[\begin{array}{c}
\left(h_{i+1,1}^{n}+h_{i+1,2}^{n}-H_{i+1}-\left(h_{i+1 / 2,2}^{n,+}-H_{i+1 / 2}\right)\right)_{+} \\
u_{i+1,1}^{n} h_{i+1 / 2,1}^{n,+} \\
\left(h_{i+1,2}^{n}-H_{i+1}+H_{i+1 / 2}\right)_{+} \\
u_{i+1,2}^{n} h_{i+1 / 2,2}^{n,+} \\
w_{1 / 2}^{n,-}= \\
q_{i+1 / 2,1}^{n,-} \\
h_{i+1 / 2,2}^{n,-} \\
q_{i+1 / 2,2}^{n,-}
\end{array}\right]=\left[\begin{array}{c}
\left(h_{i, 1}^{n}+h_{i, 2}^{n}-H_{i}-\left(h_{i+1 / 2,2}^{n,-}-H_{i+1 / 2}\right)\right)_{+} \\
u_{i, 1}^{n} h_{i+1 / 2,1}^{n,-} \\
\left(h_{i, 2}-H_{i}+H_{i+1 / 2}\right)_{+} \\
u_{i, 2}^{n} h_{i+1 / 2,2}^{n,-}
\end{array}\right],
\end{gathered}
$$

In the expressions above, the superindex + makes reference to the hydrostatic reconstruction to the right of an intercell and the index + represents the positive part of an expression.

The numerical scheme (3.1) is exactly well-balanced for the water at rest or vacuum solutions and it is able to deal with wet/dry zones. This numerical scheme will be named HR-IFCP hereafter.

\section{Numerical tests}

In this Section, the well-balanced properties, accuracy and efficiency of the numerical schemes IFCP and HR-IFCP are tested and compared with ROE and GFORCE schemes for the two layer shallow water system. Here, Roe scheme is used together with the Harten-Hyman entropy-fix technique (see [20]).

The following general facts have been observed: 
- GFORCE scheme is the most diffusive one.

- No significant differences can be found between IFCP and HR-IFCP, but IFCP gives in general slightly better results. Nevertheless, HR-IFCP has better positivity properties: in some cases as in Test 3, a significant reduction of the CFL parameter is required for IFCP in order to avoid the appearance of negative values of the thickness while this is not the case for HR-IFCP. The same conclusions are valid for ROE and HR-ROE who is the numerical scheme obtained by combining the Roe method with the hydrostatic reconstruction technique.

- No significant differences can be found between ROE and IFCP, but ROE is a bit less diffusive.

- IFCP and GFORCE are about 3 and 3.2 times faster than ROE respectively.

\subsection{Test 1: An internal dam-break problem}

This test is designed to evaluate the accuracy of the schemes for non-regular timedependent solutions over a flat bottom. The axis of the channel is the interval $[0,10]$.

The initial condition is $q_{1}(x, 0)=q_{2}(x, 0)=0$,

$$
h_{1}(x, 0)= \begin{cases}0.2, & \text { if } x<5, \\ 0.8, & \text { otherwise }\end{cases}
$$

and

$$
h_{2}(x, 0)= \begin{cases}0.8, & \text { if } x<5, \\ 0.2, & \text { otherwise }\end{cases}
$$

Free boundary conditions are considered. The CFL parameter is set to 0.9 and $r=0.98$. A reference solution is computed with ROE using a mesh of 3200 points.

Figure 2 shows the comparison of the numerical results obtained with ROE, GFORCE, and IFCP with the reference solution at time $t=10 s$ taking $\Delta x=1 / 20$. Note that no significant differences can be observed between ROE and IFCP scheme, while GFORCE is very diffusive.

The numerical results of this test seem to show that the numerical solutions provided by the different numerical schemes converge to the same solution, but this is not true: there are slight differences between the speed and the limit states of the shocks for the limits of the solutions obtained with the different schemes as $\Delta x$ tends to 0 . Nevertheless, a finer analysis is required to notice this fact (see [11]). 


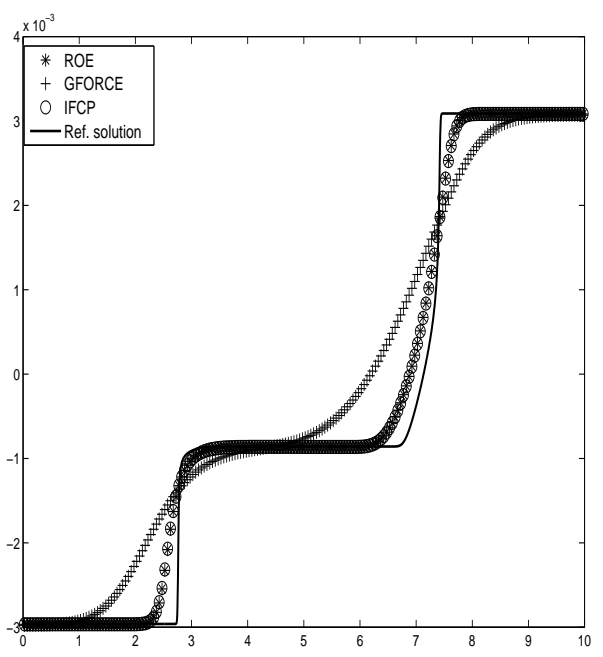

(a) Free surface

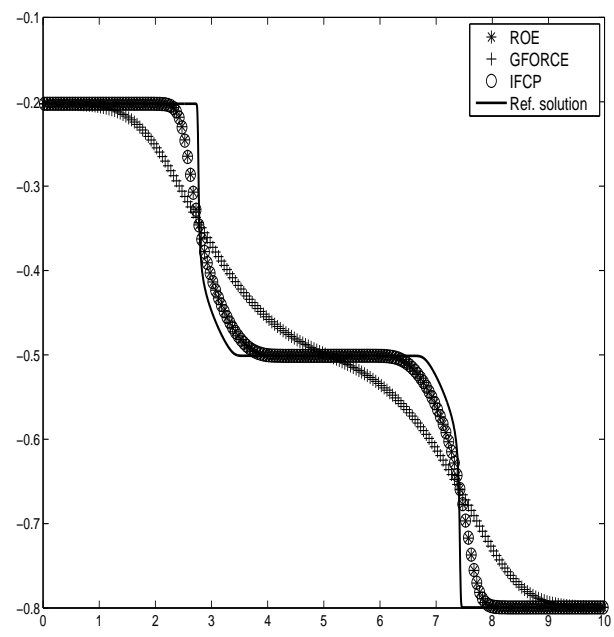

(c) Interface.

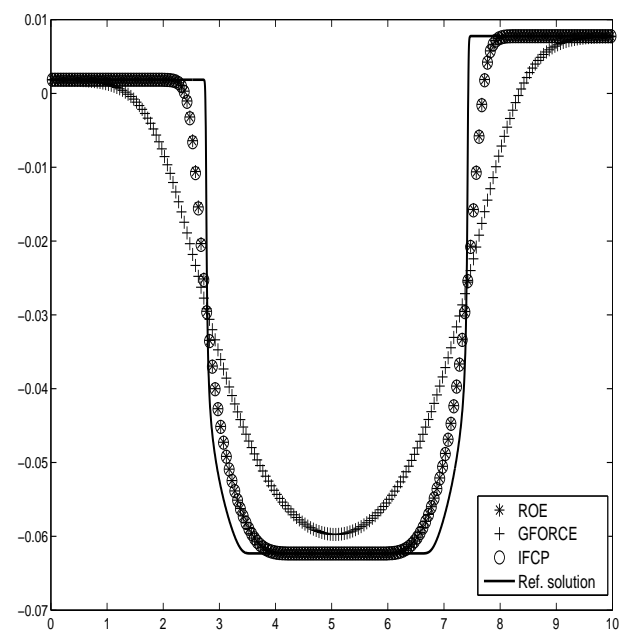

(b) Discharge. First layer.

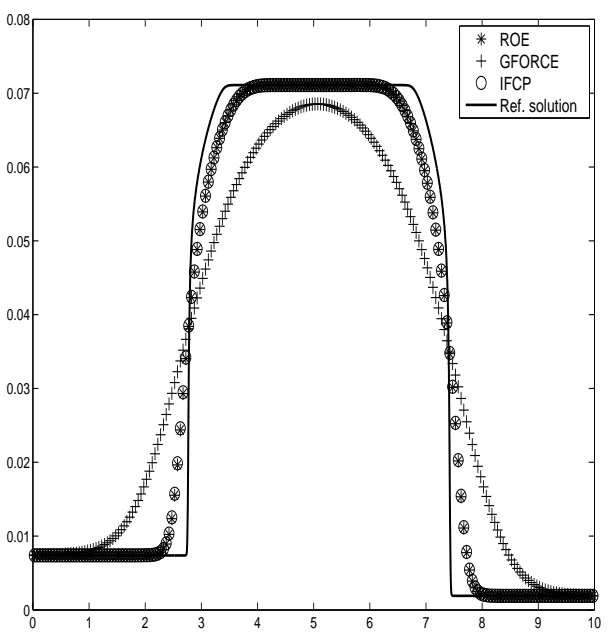

(d) Discharge. Second layer

Figure 2: Test 1: Comparison of ROE, GFORCE and IFCP with the reference solution at $t=10 \mathrm{~s}$ with $\Delta x=1 / 20$. 


\subsection{Test 2: A Riemann problem with flat bottom}

We consider the test case introduced in [12] consisting of a two-layer flow through a rectangular channel with flat bottom topography whose axis is the interval $[0,100]$. The initial condition is given by:

$$
\begin{gathered}
h_{1}(x, 0)=\left\{\begin{array}{ll}
0.5, & \text { if } x<50, \\
0.55, & \text { otherwise },
\end{array} \quad h_{2}(x, 0)= \begin{cases}0.5, & \text { if } x<5, \\
0.45, & \text { otherwise },\end{cases} \right. \\
q_{1}(x, 0)=\left\{\begin{array}{ll}
1.25, & \text { if } x<50, \\
1.375, & \text { otherwise, }
\end{array} \quad h_{2}(x, 0)= \begin{cases}1.25, & \text { if } x<5, \\
1.125, & \text { otherwise },\end{cases} \right.
\end{gathered}
$$

Free boundary conditions are considered. The CFL parameter is set to 0.9 and $r=0.98$. A reference solution is computed with ROE using a mesh of 6400 points.

Figure 3 shows the comparison of the numerical results obtained with ROE, GFORCE, and IFCP with the reference solution at time $t=5 s$ taking $\Delta x=0.125$. Note that no significant differences can be observed between ROE and IFCP scheme, while GFORCE is a bit more diffusive. In fact, GFORCE behaves better in this test case than in the previous one due to the fact that the differences between the external and the internal eigenvalues are lower.

\subsection{Test3: A Riemann problem with a bottom step}

Let us consider a rectangular channel with discontinuous topography. The axis of the channel is the interval $[0,10]$ and the bottom topography is given by

$$
H(x)=h_{1}(x, 0)= \begin{cases}1.0, & \text { if } x<5 \\ 1.5, & \text { otherwise. }\end{cases}
$$

The initial condition is $q_{1}(x, 0)=0$,

$$
h_{2}(x, 0)=\left\{\begin{array}{ll}
0.2, & \text { if } x<5, \\
0.1, & \text { otherwise },
\end{array} \quad h_{1}(x, 0)=H(x)-h_{2}(x, 0)\right.
$$

and

$$
q_{2}(x, 0)= \begin{cases}0.02, & \text { if } x<5, \\ 0.01, & \text { otherwise. }\end{cases}
$$

Free boundary conditions are considered. The ratio of densities is set to $r=0.98$ and the CFL parameter is set to 0.9 for HR-IFCP scheme and HR-ROE, and 0.3 


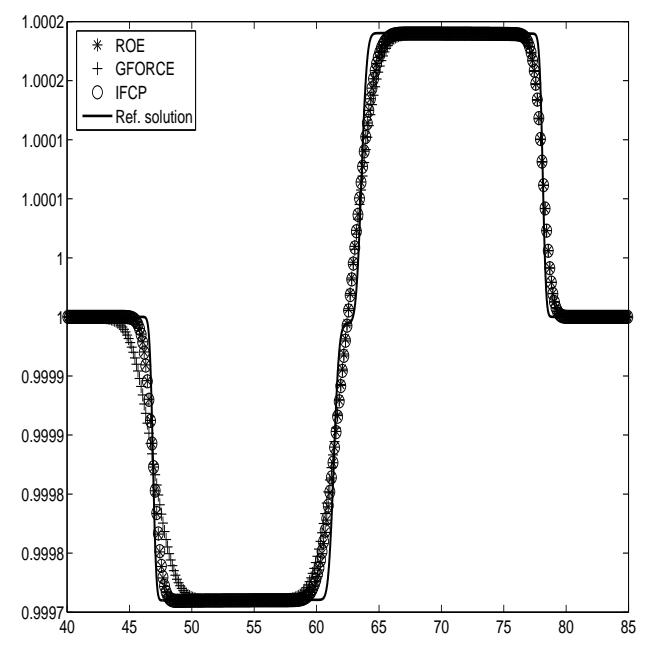

(a) Free surface

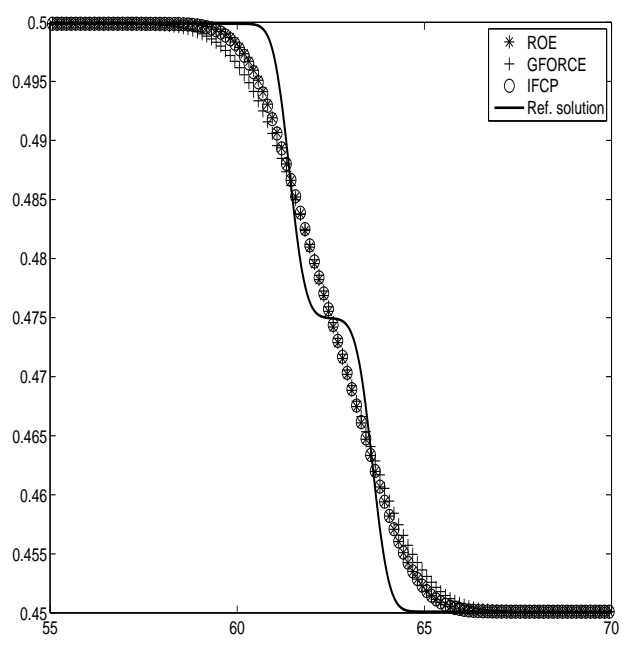

(c) Interface.

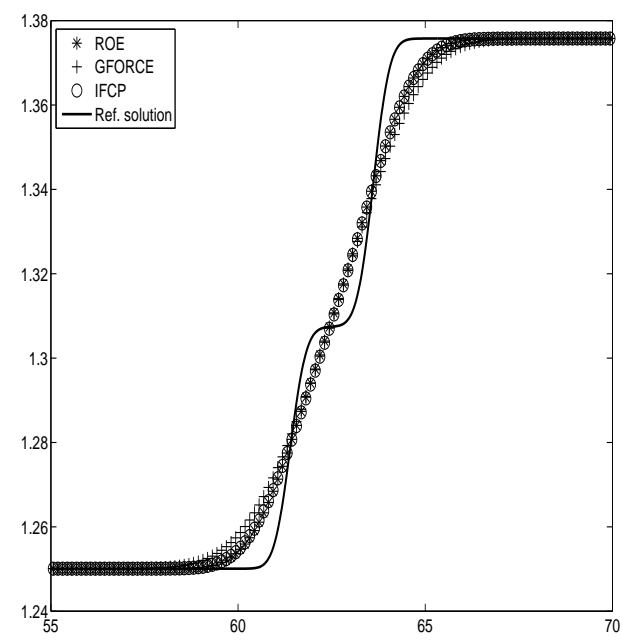

(b) Discharge. First layer.

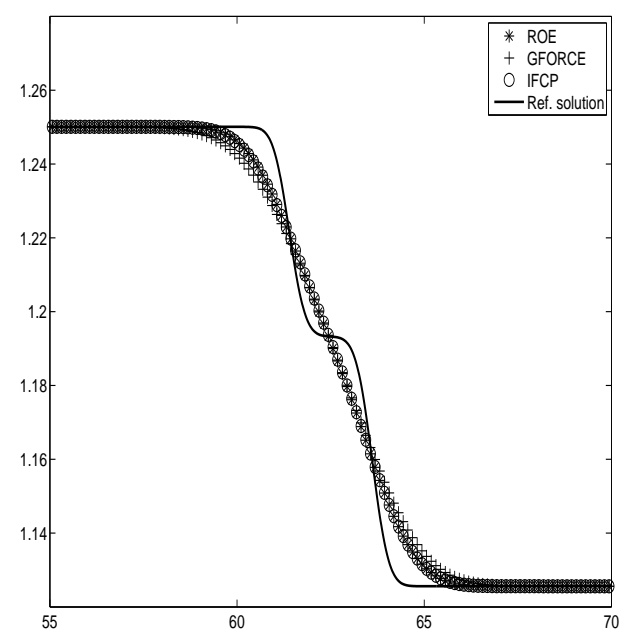

(d) Discharge. Second layer

Figure 3: Test 2: Comparison of ROE, GFORCE and IFCP with the reference solution at $t=5 \mathrm{~s}$ with $\Delta x=0.125$. 
for the IFCP scheme. This CFL reduction is needed to avoid the appearance of negative values of $h_{2}$.

A reference solution is computed with HR-ROE using a mesh of 3200 points. Figure 4 shows the comparison of the numerical results obtained with IFCP and HR-IFCP with the reference solution at time $t=2 s$ taking $\Delta x=0.05$.



(a) Interface



(b) Discharge. Second layer.

Figure 4: Test 3: Comparison of IFCP and HR-IFCP with the reference solution at $t=2 \mathrm{~s}$ with $\Delta x=0.05$.

\subsection{Test 4: Well-balanced property for a non-smooth solu- tion}

In this test the axis of the channel is again the interval $[0,10]$. The bottom topography is given by the function

$$
H(x)=1.0-e^{-(x-5.0)^{2}} .
$$

The initial condition is $q_{1}(x, 0)=q_{2}(x, 0)=0$,

$$
h_{1}(x, 0)= \begin{cases}1.0 & \text { if } x<5, \\ 0.0 & \text { otherwise }\end{cases}
$$

and

$$
h_{2}(x, 0)= \begin{cases}1.0-e^{-(x-5)^{2}} & \text { if } x<5, \\ 2.0-e^{-(x-5)^{2}} & \text { otherwise. }\end{cases}
$$

As boundary conditions, the relation $q_{1}(\cdot, t)=-q_{2}(\cdot, t)$ is imposed at $x=0$, and the free surface is fixed to $z=1$ at $x=10$, that is $h_{1}(10, t)+h_{2}(10, t)-H(x)=1.0$. The CFL parameter is set to 0.9 and $r=0.98$. 
Table 1: Test 4: CPU time (in seconds).

\begin{tabular}{||c||c|c|c|c||}
\hline N. Cells & ROE & GFORCE & IFCP & HR-IFCP \\
\hline \hline 50 & 15.46 & 5.09 & 5.28 & 5.45 \\
100 & 61.79 & 19.39 & 20.35 & 21.04 \\
200 & 245.36 & 77.21 & 81.00 & 83.75 \\
400 & 990.70 & 309.60 & 324.82 & 335.83 \\
\hline
\end{tabular}

A reference solution is computed with a high order Roe scheme using a mesh of 800 points. Figure 5 shows the comparison of ROE, GFORCE, IFCP, and HR-IFCP with the reference solution (only the interface and the discharge of the first layer are shown) at time $t=300$ taking $\Delta x=1 / 20$. Note that while GFORCE is very diffusive, IFCP captures quite well the internal stationary bore.

Observe that the results obtained with IFCP are slightly better than those obtained with HR-IFCP. The same conclusion is drawn if the numeric results obtained with Roe are compared with those obtained by combining the Roe method with the hydrostatic reconstruction technique (HR-ROE hereafter): compare the results obtained with HR-ROE and HR-IFCP shown in the Figure 6 with those obtained with ROE and IFCP in Figure 5.

Table 1 summarises the CPU time (in seconds) for the different numerical schemes. Note that GFORCE is about 3.2 times faster than ROE scheme, nevertheless, the result obtained by GFORCE is poor in comparison with IFCP, being the CPU cost of the same order. Observe that HR-IFCP is $5 \%$ more expensive than IFCP, while IFCP is a bit more accurate than HR-IFCP.

The stationary solution of this test problem exhibits a transonic rarefaction located at the point of minimal depth. Observe that both the IFCP or HR-IFCP methods capture correctly this rarefaction, even though no entropy-fix technique is added.

\subsection{Test 5: Lock-exchange solution}

In [18] Farmer \& Army (F\&A hereafter) computed some approximated exchange flows through channels with rectangular cross-sections and a single bump by means of a simplified rigid-lid stationary model in which the density ratio is supposed to be close to 1 . The aim of this test is to compare the results provided by ROE and ICFP schemes against these approximated stationary solutions. To do this, the classical lock exchange experiment is considered: the two layer of fluids are initially at rest and put apart by an artificial dam located at the crest of the bump (see Figure $7(\mathrm{a}))$. The dam is removed at time $t=0$ and the layer are let to evolve until a stationary state is reached. In this particular test case, the bottom topography is 


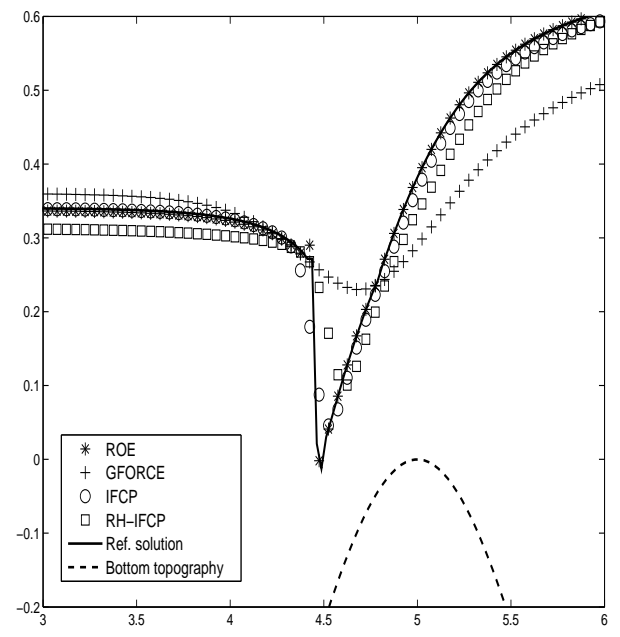

(a) Interface (zoom)

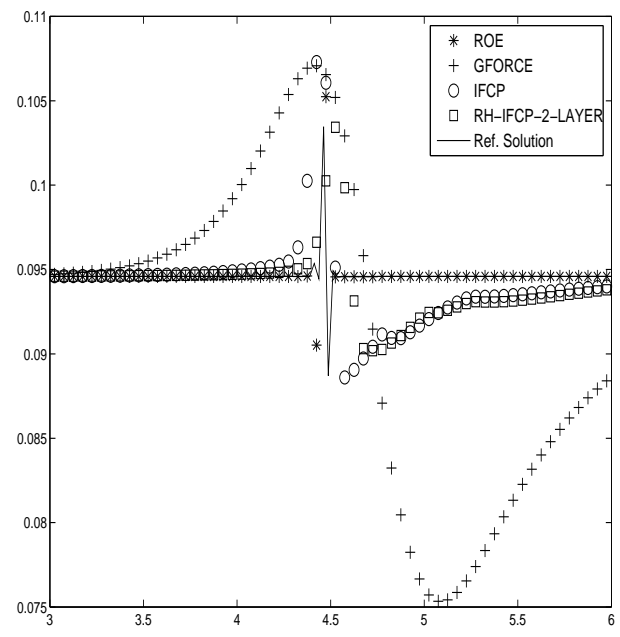

(b) Discharge (zoom). First layer

Figure 5: Test 4: Comparison of ROE, GFORCE, IFCP and HR-IFCP with the reference solution at time $t=300$ with $\Delta x=1 / 20$.

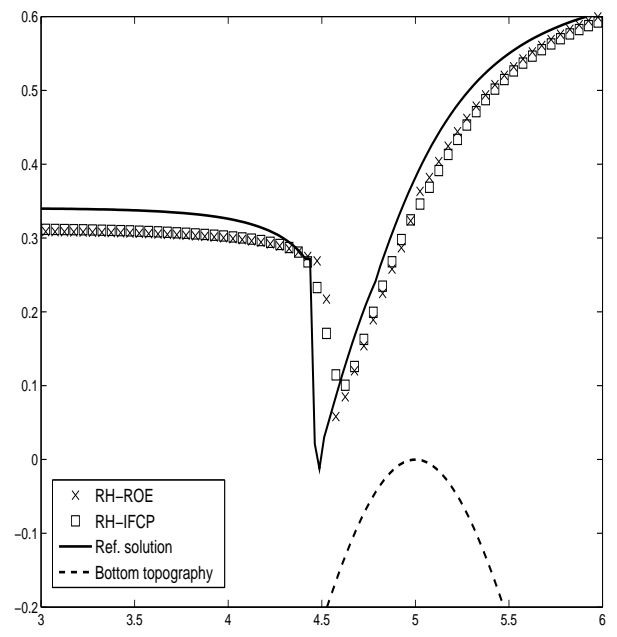

(a) Interface (zoom)

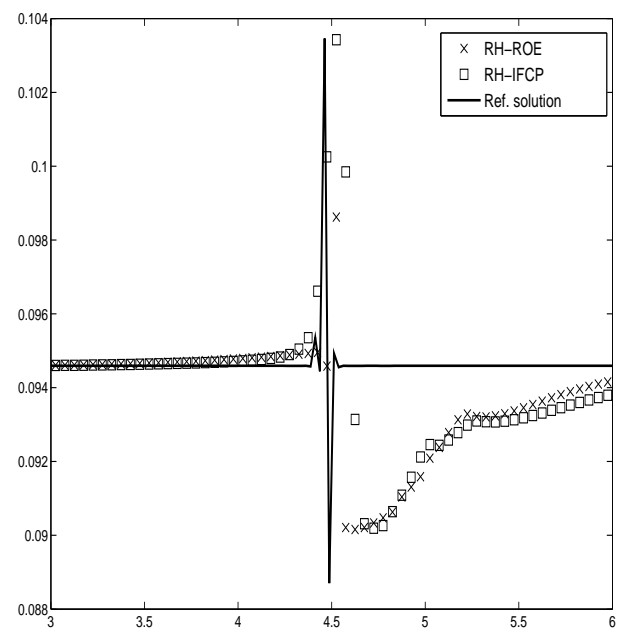

(b) Discharge (zoom). First layer

Figure 6: Test 4: Comparison of HR-ROE and HR-IFCP with the reference solution at time $t=300$ with $\Delta x=1 / 20$. 
defined by the function $H(x)=2-e^{-x^{2}}, x \in[-3,3]$. As boundary conditions, only the condition $q_{1}=-q_{2}$ is imposed at both ends. The ratio of densities is set to 0.98 , the CFL parameter is set to 0.9 , and $\Delta x=0.03$. Figure $7(\mathrm{~b})$ shows the interface corresponding to the stationary solutions reached with both schemes and they are compared with the corresponding to the F\&A solution. Note that ROE and IFCP schemes give similar results which are also close to the F\&A solution. In fact, only some small differences appear in the right end of the channel which are due mainly to the rigid lid approximation in the $\mathrm{F} \& \mathrm{~A}$ model. The exchange flux computed are $q_{1}=-q_{2}=9.366 \cdot 10^{-2} \mathrm{~m}^{3} / \mathrm{s}$ for the ROE scheme, $q_{1}=-q_{2}=9.406 \cdot 10^{-2} \mathrm{~m}^{3} / \mathrm{s}$ for the IFCP scheme, while $F \& A$ model gives $q_{1}=-q_{2}=9.213 \cdot 10^{-2} \mathrm{~m}^{3} / \mathrm{s}$.

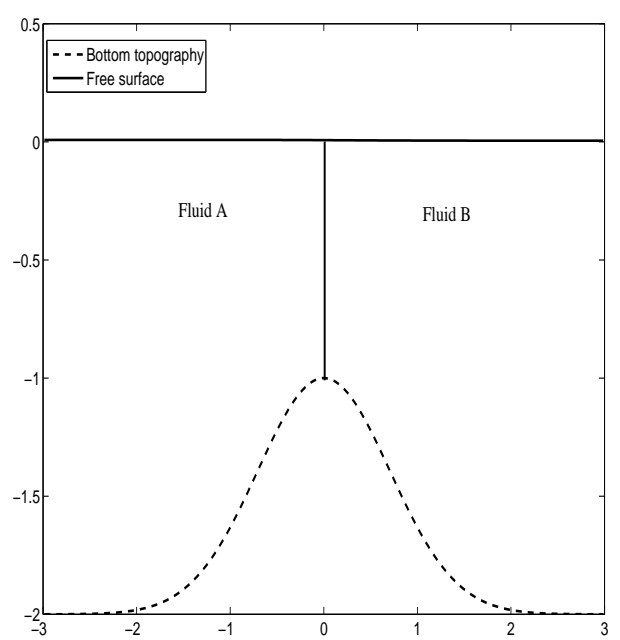

(a) Initial condition

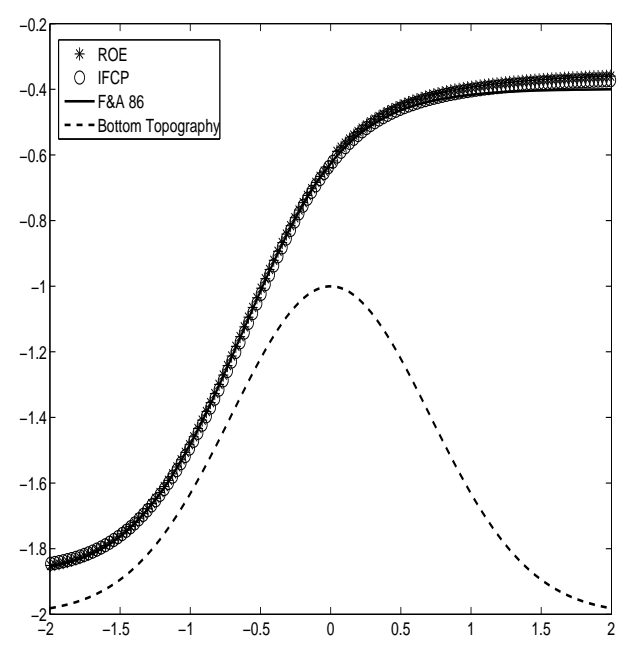

(b) Interface (stationary state)

Figure 7: Test 5: Initial condition and comparison of ROE and IFCP scheme with F\&A stationary solution with $\Delta x=0.03$.

\subsection{Test 6: Wet/dry front over an irregular bottom topog- raphy}

In this test, the axis of the channel is again the interval $[0,10]$. The bottom topography is given by the function

$$
H(x)= \begin{cases}0.5 & \text { if } x<4 \\ 1.0 & \text { otherwise }\end{cases}
$$

The initial condition is $q_{1}(x, 0)=q_{2}(x, 0)=0$,

$$
h_{2}(x, 0)= \begin{cases}0.0 & \text { if } x<4, \\ 0.8 & \text { if } 4.5<x<5.0 \\ 0.2 & \text { otherwise }\end{cases}
$$


and

$$
h_{1}(x, 0)=H(x)-h_{2}(x, 0)+0.5 .
$$

Free boundary conditions are imposed. The CFL parameter is set to 0.8 and $r=0.99$.

Figure 8 shows the evolution of the free surface computed with ROE and IFCP. We compare the solutions given by both schemes with a reference solution computed with ROE with a mesh of 3200 points. Note that both schemes provide solutions of the same quality, being IFCP more precise in the area located at the top of the step (see Figures 8(c)-8(f)). Figure 9 shows the evolution of the discharge of the second layer for both schemes at the same times. Again, both schemes give solutions of a similar quality. ROE is a bit less diffusive than IFCP (see Figures 9(e)-9(f)), but IFCP is more accurate in the area located at the top of the step. The robustness of IFCP scheme is assessed with this very difficult numerical test.

\subsection{Test 7: Wet/dry front over a smooth bottom topogra- phy}

In this test, the axis of the channel is again the interval $[0,10]$. The bottom topography is given by the function

$$
H(x)= \begin{cases}1 & \text { if } x \leq 5 \\ 1-\frac{x-5}{10} & \text { otherwise. }\end{cases}
$$

The initial condition is $q_{1}(x, 0)=q_{2}(x, 0)=0$,

$$
h_{2}(x, 0)= \begin{cases}H(x)-0.4 & \text { if } 4.5<x<5.0 \\ 0.0 & \text { if } x>7 \\ H(x)-0.8 & \text { otherwise }\end{cases}
$$

and

$$
h_{1}(x, 0)=H(x)-h_{2}(x, 0) .
$$

Free boundary conditions are imposed. The CFL parameter is set to 0.8 and $r=$ 0.99 .

Figure 10 shows the evolution of the free surface computed with ROE and IFCP. We compare the solutions given for both schemes with a reference solution computed with ROE scheme using a mesh with 3200 cells. Observe that again both schemes provide solutions of the same quality, being ROE a bit more precise.

\section{Conclusions}

In this article the new first order numerical scheme ICFP for solving the two-layer shallow water system has been introduced. This method is based on a suitable decomposition of a Roe matrix $A_{\Phi}\left(w_{L}, w_{R}\right)$ that uses a viscosity matrix which is 


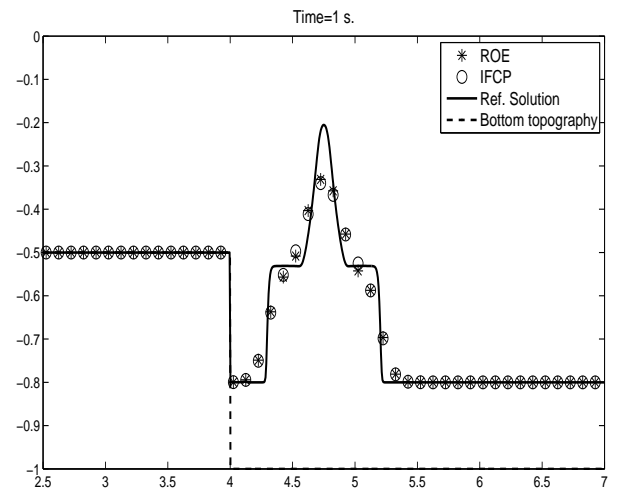

(a) $\mathrm{t}=1.0 \mathrm{~s}$.

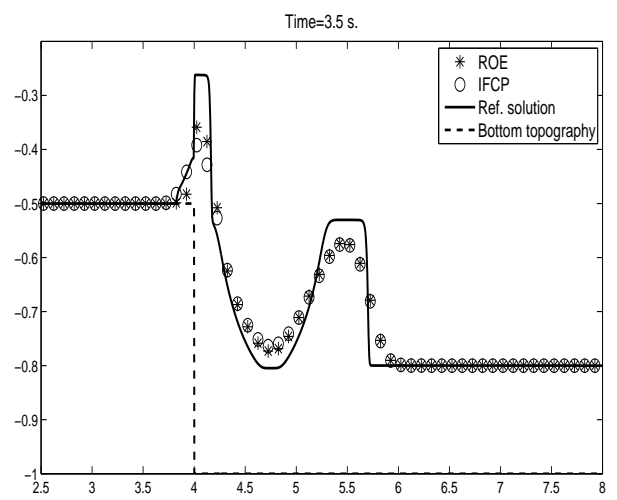

(c) $\mathrm{t}=3.5 \mathrm{~s}$.



(e) $\mathrm{t}=7.0 \mathrm{~s}$.

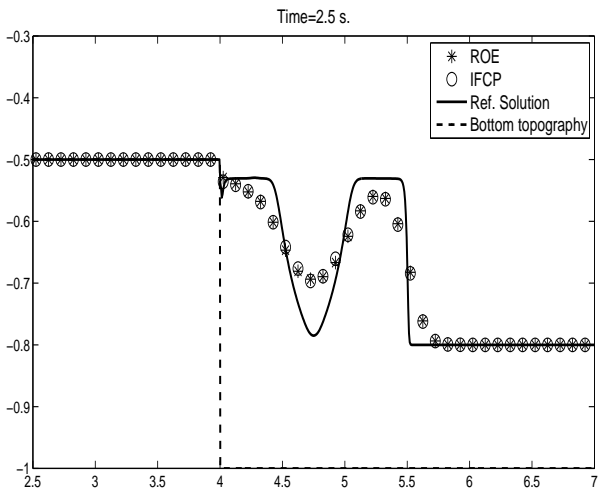

(b) $\mathrm{t}=2.5 \mathrm{~s}$.

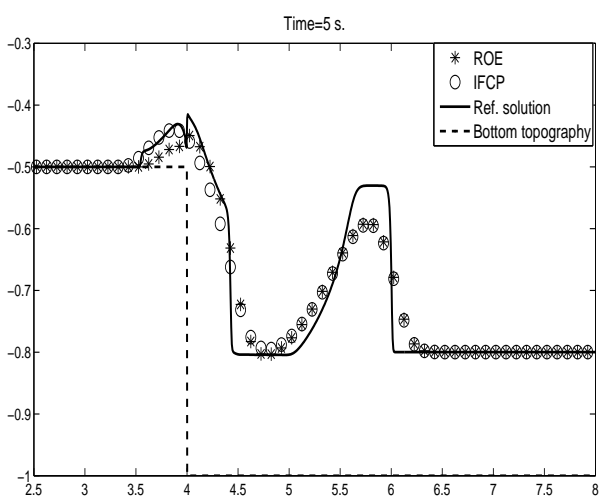

(d) $\mathrm{t}=5.0 \mathrm{~s}$.

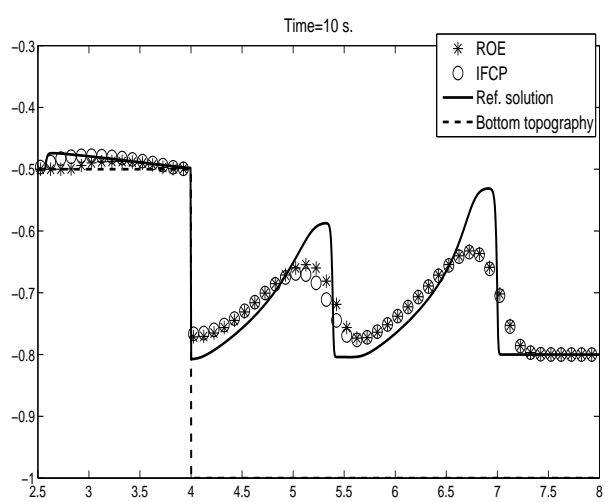

(f) $\mathrm{t}=10.0 \mathrm{~s}$.

Figure 8: Test 6 (interface): Comparison of ROE and IFCP with the reference solution at different times with $\Delta x=1 / 20$. 


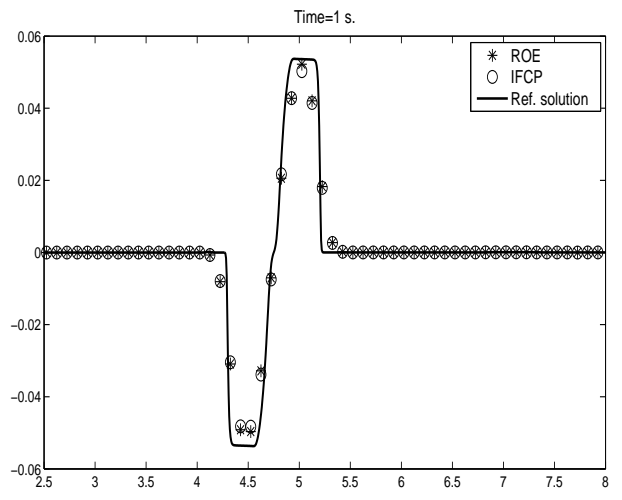

(a) $\mathrm{t}=1.0 \mathrm{~s}$.

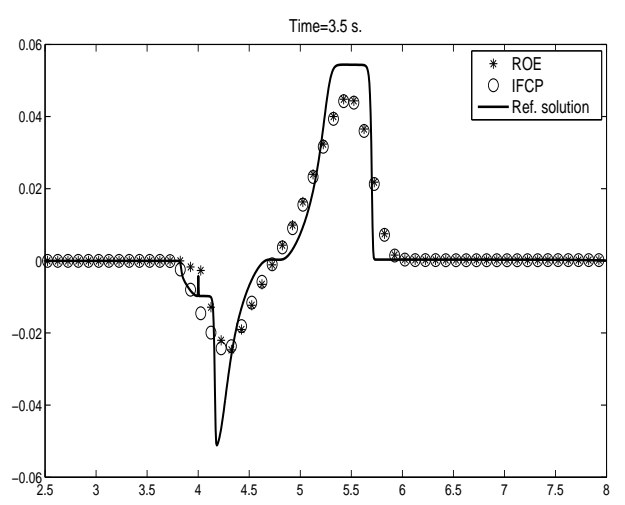

(c) $\mathrm{t}=3.5 \mathrm{~s}$.

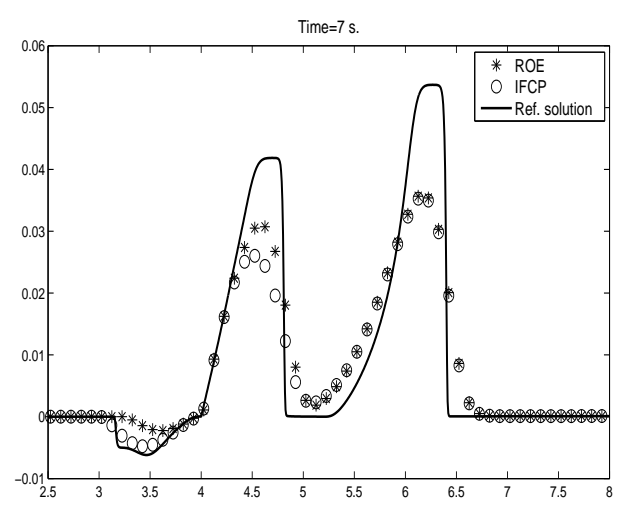

(e) $\mathrm{t}=7.0 \mathrm{~s}$.

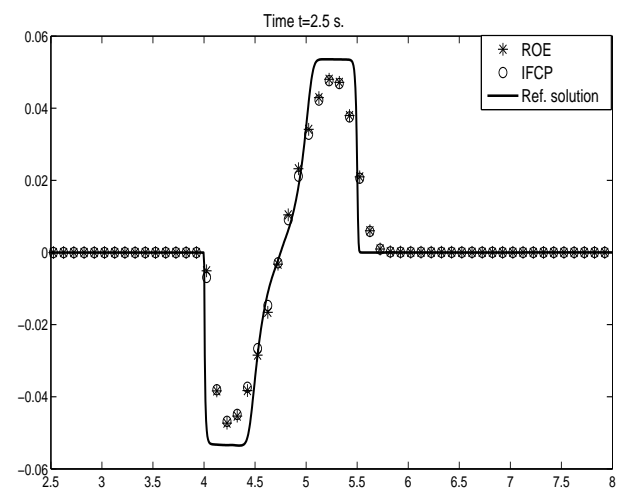

(b) $\mathrm{t}=2.5 \mathrm{~s}$.

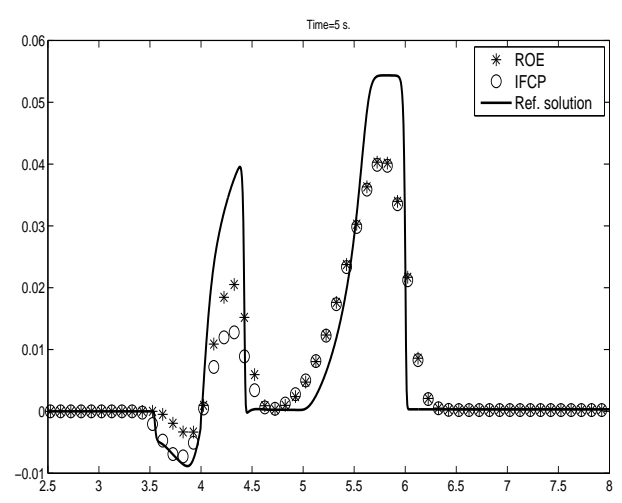

(d) $\mathrm{t}=5.0 \mathrm{~s}$.

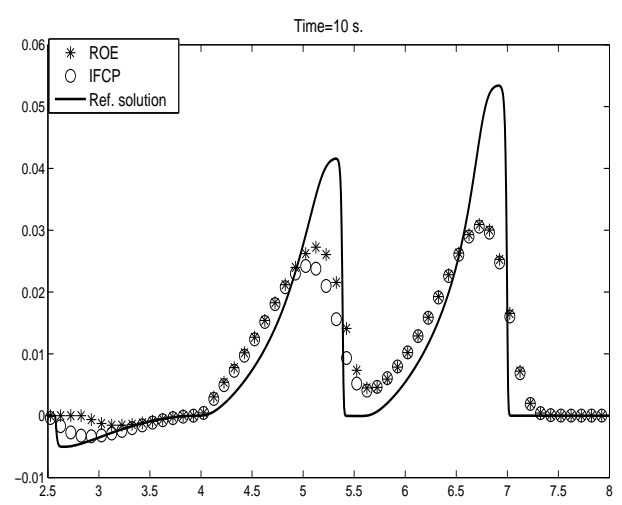

(f) $\mathrm{t}=10.0 \mathrm{~s}$.

Figure 9: Test 6 (discharge of the second layer): Comparison of ROE and IFCP with the reference solution at different times with $\Delta x=1 / 20$. 


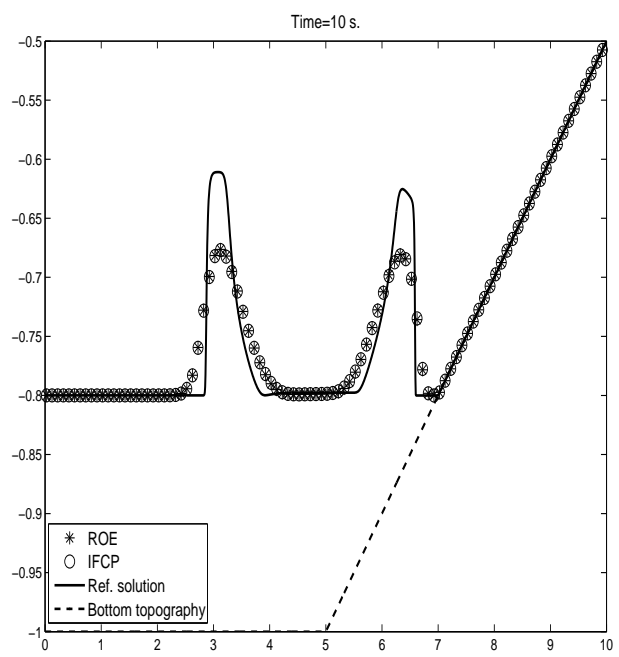

(a) $\mathrm{t}=1.0 \mathrm{~s}$.

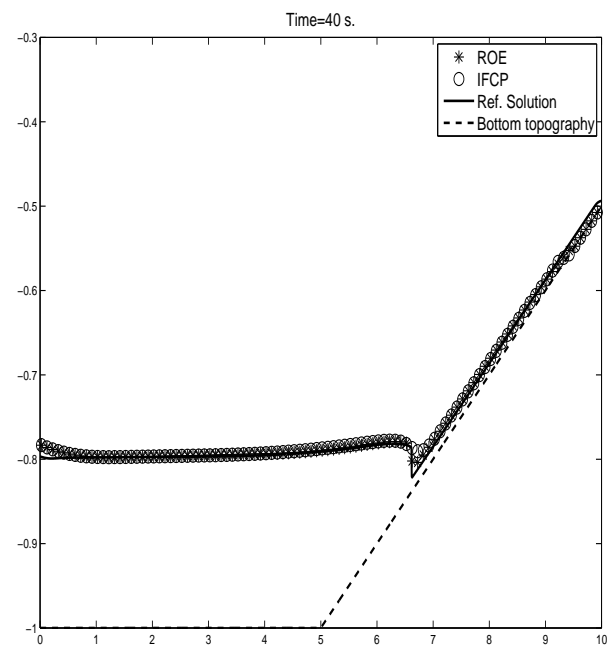

(c) $\mathrm{t}=3.5 \mathrm{~s}$.

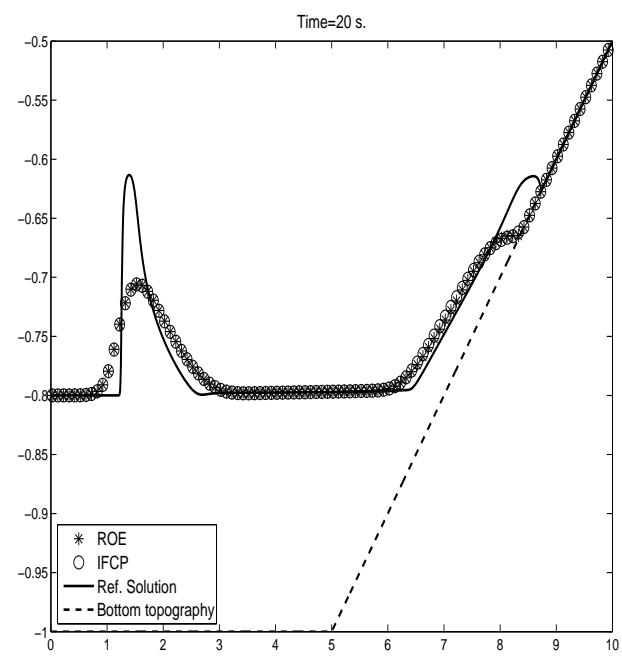

(b) $\mathrm{t}=2.5 \mathrm{~s}$.



(d) $\mathrm{t}=5.0 \mathrm{~s}$.

Figure 10: Test 7 (interface): Comparison of ROE and IFCP with the reference solution at different times with $\Delta x=1 / 20$. 
a linear combination of the identity matrix, $A_{\Phi}\left(w_{L}, w_{R}\right)$, and $A_{\Phi}^{2}\left(w_{L}, w_{R}\right)$ whose coefficients are computed in terms of the eigenvalues of $A_{\Phi}\left(w_{L}, w_{R}\right)$. The resulting numerical scheme is linearly $L_{\infty}$-stable for the two-layer shallow water system. In practice, some known first order approximations of the eigenvalues can be used to define the coefficients of the viscosity matrix what reduces dramatically the computational cost. The well-balanced property of the numerical scheme has been also studied. Finally, some numerical tests have been performed to check the efficiency and the good properties of the numerical scheme. The numerical tests show that the results obtained with IFCP are close to those obtained with ROE scheme and much better than GFORCE scheme. Concerning the CPU time, IFCP is similar to GFORCE and about 3 times faster than ROE scheme. Moreover, no entropy-fix technique is required.

The extension to high order and to two-dimensional problems is straightforward following [8] and [9].

Even though IFCP is designed for the two-layer shallow water system, it can be easily extended to some other nonconservative hyperbolic systems, like the twophase fluid model proposed in [25] or the submarine avalanches model proposed in [19] for example.

\section{Acknowledgments}

This research has been partially supported by the Spanish Government Research projects MTM2009-07719, MTM2009-11923 and P06-RNM-01594. The numerical computations have been performed at the Laboratory of Numerical Methods of the University of Málaga. 


\section{Appendix}

Let us prove that the numerical scheme defined by (2.14)-(2.16), where $Q_{\Phi}\left(w_{L}, w_{R}\right)$ is given by (2.21)-(2.25) is linearly $L^{\infty}$-stable for the two-layer shallow water equations under the usual CFL condition. We suppose that

$$
\lambda_{1}<0, \quad \lambda_{4}>0, \quad\left|\lambda_{l}\right|<\left|\lambda_{1}\right|, \quad\left|\lambda_{l}\right|<\left|\lambda_{4}\right|, \quad l=2,3,
$$

being $\lambda_{j}, j=1, \cdots, 4$, the eigenvalues of the matrix $A_{\Phi}$. These conditions over the eigenvalues are commonly satisfied for two-layer geophysical flows. Let us denote by $\lambda_{Q, j}, j=1, \cdots, 4$, the four eigenvalues of the matrix $Q_{\Phi}$ defined by $(2.21)$. Note that

$$
\lambda_{Q, j}=p\left(\lambda_{j}\right)=\alpha_{0}+\alpha_{1} \lambda_{j}+\alpha_{2} \lambda_{j}^{2}, \quad j=1, \ldots, 4 .
$$

Let us prove that $\lambda_{Q, j} \geq\left|\lambda_{j}\right|$. It is clear for construction that $\lambda_{Q, j}=\left|\lambda_{j}\right|, j=1,4$. Thus, we must prove that $\lambda_{Q, j} \geq\left|\lambda_{j}\right|, j=2,3$. As $\lambda_{j} \in\left[-\left|\chi_{i n t}\right|,\left|\chi_{i n t}\right|\right], j=2,3$, then, it is enough to prove that $p(\lambda) \geq|\lambda|$ for $\lambda \in\left[-\left|\chi_{\text {int }}\right|,\left|\chi_{\text {int }}\right|\right]$.

Let us prove first that $\alpha_{2}>0$. To do that, let us rewrite $\alpha_{2}$ as $\alpha_{2}=\beta \gamma_{2}$ with

$$
\begin{gathered}
\beta=\frac{1}{\left(\lambda_{1}-\chi_{i n t}\right)\left(\lambda_{1}-\lambda_{4}\right)\left(\chi_{i n t}-\lambda_{4}\right)}, \\
\gamma_{2}=\left|\lambda_{1}\right|\left(\chi_{i n t}-\lambda_{4}\right)+\left|\chi_{i n t}\right|\left(\lambda_{4}-\lambda_{1}\right)+\left|\lambda_{4}\right|\left(\lambda_{1}-\chi_{i n t}\right) .
\end{gathered}
$$

Taking into account the definition of $\chi_{\text {int }}$ and (5.1), it is clear that $\left(\lambda_{1}-\chi_{\text {int }}\right)<0$ and $\left(\chi_{\text {int }}-\lambda_{4}\right)<0,\left|\chi_{\text {int }}\right|<\left|\lambda_{1}\right|$ and $\left|\chi_{\text {int }}\right|<\left|\lambda_{4}\right|$. Then, $\beta<0$. Moreover,

$$
\left|\chi_{i n t}\right|\left(\lambda_{1}-\lambda_{4}\right)=\left|\chi_{i n t}\right|\left(\lambda_{1}-\chi_{i n t}\right)+\left|\chi_{i n t}\right|\left(\chi_{i n t}-\lambda_{4}\right),
$$

therefore,

$$
\left|\chi_{i n t}\right|\left(\lambda_{1}-\lambda_{4}\right)>\left|\lambda_{1}\right|\left(\lambda_{1}-\chi_{i n t}\right)+\left|\lambda_{4}\right|\left(\chi_{i n t}-\lambda_{4}\right) .
$$

Therefore $\gamma_{2}<0$ and then $\alpha_{2}>0$.

Let us now prove that $\operatorname{sgn}\left(\alpha_{1}\right)=-\mathcal{S}_{\text {ext }}$, where $\alpha_{1}$ is defined by (2.25). As in the previous case, $\alpha_{1}=-\beta \gamma_{1}$, where $\beta<0$ is defined by (5.2) and

$$
\gamma_{1}=\left(\left|\lambda_{1}\right|-\left|\lambda_{4}\right|\right)\left(\left|\lambda_{1}\right|-\left|\chi_{\text {int }}\right|\right)\left(\left|\lambda_{4}\right|-\left|\chi_{\text {int }}\right|\right)
$$

Taking into account (5.1) and the definition of $\mathcal{S}_{\text {ext }}(2.24)$, we obtain $\operatorname{sgn}\left(\alpha_{1}\right)=$ $-\mathcal{S}_{\text {ext }}$.

The key point in the definition of $\chi_{i n t}$ is $\mathcal{S}_{\text {ext }}$, as the sign of $\alpha_{1}$ depends on the definition of $\mathcal{S}_{\text {ext }}$.

Let us suppose that $\left|\lambda_{1}\right|>\left|\lambda_{4}\right|$ (otherwise the proof is analogous), then $\mathcal{S}_{\text {ext }}=-1$ and consequently $\alpha_{1}>0$. Then, $p(\lambda) \geq p(-\lambda)$ for $\lambda>0$. So, it is enoguh to prove that

$$
p(\lambda) \geq-\lambda \quad \forall \lambda \in\left[-\left|\chi_{\text {int }}\right|, 0\right]=\left[\chi_{\text {int }}, 0\right] .
$$


Let us now define $\lambda_{v}=\left(-\alpha_{1}\right) /\left(2 \alpha_{2}\right)$, the point where $p(\lambda)$ achieves its minimum value. Note that $\lambda_{v}<0$. Two possibilities can be distinguished:

- Case A: $\lambda_{v} \leq \chi_{\text {int }}$. In this case

$$
p(\lambda)-p\left(\chi_{i n t}\right)=\left(\lambda-\chi_{i n t}\right)\left(\alpha_{1}+2 \alpha_{2} \chi_{i n t}\right)+\alpha_{2}\left(\lambda-\chi_{i n t}\right)^{2} \geq 0,
$$

as $\lambda \geq \chi_{\text {int }}$. Therefore, $p(\lambda) \geq p\left(\chi_{\text {int }}\right)=\left|\chi_{\text {int }}\right| \geq|\lambda|, \forall \lambda \in\left[\chi_{\text {int }}, 0\right]$.

- Case B: $\lambda_{v}>\chi_{i n t}$. Note that $p\left(\lambda_{1}\right)=-\lambda_{1}, p\left(\chi_{i n t}\right)=-\chi_{\text {int }}$ and, $\lambda_{1}<\chi_{\text {int }}<0$. Therefore, there is not any other value $\lambda<0$ such us $p(\lambda)=-\lambda$. Noting that $\alpha_{2}>0$ and $\lambda_{v}>\chi_{\text {int }}$ then $p(\lambda)>-\lambda, \forall 0 \geq \lambda>\chi_{\text {int }}$.

The $L^{\infty}$-stability of the scheme can be proved even if the property (5.1) fails, but the proof is not included here for the sake of shortness.

\section{References}

[1] R. Abgrall, S. KARni. Two-layer shallow water system: a relaxation approach. SIAM J. Sci. Comput. 31: 1603-1627, 2009.

[2] R. Abgrall, S. Karni. A comment on the computation of nonconservative products. Jour. Comp. Phys. 229: 27592763, 2010.

[3] E. Audusse. A multilayer Saint-Venant model: derivation and numerical validation. Discrete Contin. Dyn. Syst. Ser. B 5: 189-214, 2005.

[4] E. Audusse, F. Bouchut, M.O. Bristeau, R. Klein, B. Perthame. A fast and stable well-balanced scheme with hydrostatic reconstruction for shallow water flows. SIAM J. Sci. Comp. 25 (6): 2050-2065, 2004.

[5] E. Audusse, M.O. Bristeau. Finite-volume solvers for a multilayer Saint-Venant system. Int. J. Appl. Math. Comput. Sci. 17: 311-319, 2007.

[6] F. Bouchut, T. Morales de Luna. An entropy satisfying scheme for two-layer shallow water equations with uncoupled treatment. M2AN Math. Model. Numer. Anal. 42 : 683-698, 2008.

[7] M.J. Castro, T. Chacón, E.D. Fernández, C. Parés. On wellbalanced finite volume methods for non-conservative non-homogeneous hyperbolic systems. SIAM J. Sci. Comput. 29: 1093-1126, 2007.

[8] M.J. Castro, J.J. Gallardo, C. Parés. High order finite volume schemes based on reconstruction of states for solving hyperbolic systems with nonconservative products. Applications to shallow water systems. Math. Comp. 75:1103-1134, 2006. 
[9] M.J. Castro, E.D. Fernández, A.M.Ferreiro, J.A. García, C. PARÉs. High order extensions of Roe schemes for two dimensional nonconservative hyperbolic systems. Jour. Sci. Comp. 39: 67-114, 2009.

[10] M.J. Castro, A.M.Ferreiro, J.A. García, J.M. González, J. Macías, C. PARÉs, M.E. VÁzquez. On the numerical treatment of wet/dry fronts in shallow flows: applications to one-layer and two-layer systems. Math. Comp. Model. 42 (3-4): 419-439, 2005.

[11] M.J. Castro, P.G. LeFloch, M.L. Muñoz, C. Parés. Why many theories of shock waves are necessary: Convergence error in formally path-consistent schemes. Jour. Comp. Phys. 3227: 8107-8129, 2008.

[12] M.J. Castro, J. Macías, C. ParÉs. A Q-Scheme for a class of systems of coupled conservation laws with source term. Application to a two-layer 1-D shallow water system. Math. Mod. Num. Anal. 35(1):107-127, 2001.

[13] M.J. Castro, A. Pardo, C. Parés. Well-Balanced numerical schemes based on a generalized hydrostatic reconstruction technique. Math. Mod. Meth. App. Sci., 17: 2055-2113, 2007.

[14] M.J. Castro, A. Pardo, C. Parés, E.F. Toro. On some fast wellbalanced first order solvers for nonconservative systems. Math. Comp. 79: 1427-1472, 2010.

[15] G. Dal Maso, P.G. LeFloch, F. Murat. Definition and weak stability of nonconservative products. J. Math. Pures Appl. 74: 483548, 1995.

[16] P. Degond, P.F. Peyrard, G. Russo, Ph. Villedieu. Polynomial upwind schemes for hyperbolic systems. C. R. Acad. Sci. Paris 1 328, 479-483, 1999.

[17] M. Dumbser, M.J. Castro, C. Parés, E.F. Toro. ADER schemes on unstructured meshes for nonconservative hyperbolic systems: applications to geophysical flows. Comp.\& Fluids 38:1731-1748, 2009.

[18] D. Farmer, L. Armi Maximal two-layer exchange over a sill and through a combination of a sill and contraction with barotropic flow. J. Fluid Mech., 164:53-76,1986.

[19] E. D. Fernández-Nieto, F. Bouchut, D. Bresch, M.J. Castro, A. Mangeney. A new Savage-Hutter type model for submarine avalanches and generated tsunami. J. Comput. Phys., 227(16): 7720$7754,2008$. 
[20] A. Harten, J.M. Hyman. Self-adjusting grid methods for onedimensional hyperbolic conservation laws. J. Comp. Phys. 50: 235-269, 1983.

[21] Hou T.Y., LeFloch P.G., Why nonconservative schemes converge to wrong solutions: error analysis. Math. Comp. 62: 497-530, 1994.

[22] A. Kurganov, G. Petrova. Central-upwind schemes for two-layer shallow water equations. SIAM J. Sci. Comput. 31: 1742-1773, 2009.

[23] C. PARÉs. Numerical methods for nonconservative hyperbolic systems: a theoretical framework. SIAM J. Num. Anal. 44(1): 300-321, 2006.

[24] C. Parés, M.J. Castro. On the well-balance property of Roes method for nonconservative hyperbolic systems. Applications to shallow water Systems. M2AN, Vol. 38, N5, pp. 821-852, 2004.

[25] E.B. Pitman, L. Le. A two-fluid model for avalanche and debris flows. Phil. Trans. R. Soc. A 363:1573-1601, 2005.

[26] J.B. Schijf, J.C. Schonfeld. Theoretical considerations on the motion of salt and fresh water. In Proc. of the Minn. Int. Hydraulics Conv., 321-333. Joint meeting IAHR and Hyd. Div. ASCE., Sept. 1953.

[27] I. Toumi. A weak formulation of Roe approximate Riemann solver. J. Comp. Phys. 102(2): 360-373, 1992. 